\title{
Stochastic Reduced-Order Model in Low-Frequency Dynamics in Presence of Numerous Local Elastic Modes
}

\author{
C. Soize \\ Professor, \\ Laboratoire Modélisation et Simulation Multi-Echelle \\ MSME UMR 8208 CNRS \\ Université Paris-Est \\ 5 bd Descartes, 77454 Marne-la-Valle, France \\ Email: christian.soize@univ-paris-est.fr \\ A. Batou \\ Assistant Professor, \\ Laboratoire Modélisation et Simulation Multi-Echelle \\ MSME UMR 8208 CNRS \\ Université Paris-Est \\ 5 bd Descartes, 77454 Marne-la-Valle, France \\ Email: anas.batou@univ-paris-est.fr
}

\begin{abstract}
This paper deals with the non usual case in structural dynamics for which a complex structure exhibits both the usual global elastic modes and numerous local elastic modes in the low-frequency range. Despite the presence of these local elastic modes, we are interested in constructing a stochastic reduced-order model using only the global modes and in taking into account the local elastic modes with a probabilistic approach. In a first part, a formulation and an algorithm which allow the "global elastic modes" and the "local elastic modes" to be calculated, are presented. The second part is devoted to the construction of the stochastic reduced-order model with the global elastic modes and in taking into account the uncertainties on the effects of the local elastic modes by the nonparametric probabilistic approach. Finally, an application which validates the theory proposed is presented.
\end{abstract}




\section{Introduction}

Linearized vibrations of conservative structures and structural modes (or elastic modes) play an important role to analyze with computational models the responses of complex structures in the low-frequency range for which the modal analysis is a very efficient and effective tool. This low-frequency range has been extensively studied and can be found in many structural vibration textbooks ( [1-6]) for fundamental bases, in [5-13] for dynamic substructuring techniques and, for instance, in $[14,15]$ for dynamic systems control.

In general, the low-frequency range (for which the modal density is very small and for which the resonances are isolated) can clearly be separated from the medium-frequency range (for which the modal density is larger but not uniform in frequency [6]). In this low-frequency range, the first elastic modes are global modes while, in the medium-frequency range, the elastic modes are mainly made up of numerous elastic modes which are more or less local. In the low-frequency range, the dynamical analysis of the structure is carried out using the first global elastic modes which are calculated with a computational model and a reduced-order model is constructed with the modal analysis in order to calculate the dynamical responses. This frequency band of analysis is not really sensitive to uncertainties in the computational model due to the robustness of the global elastic modes with respect to uncertainties.

Sometimes, a complex structure for which the low-frequency range must be analyzed as explained above, can exhibit both the global elastic modes (which characterize this low-frequency range) and numerous local elastic modes in this lowfrequency range. This situation appears for complex heterogeneous structures presenting stiff parts and flexible parts such as an automotive vehicle. Nevertheless, in such a case, the engineers are still interested in constructing a reduced-order model for which only the global elastic modes are used in order to dispose of an adapted model to carry out an optimal design under constraints defined by dynamics targets. This means that local elastic modes which are not really significant in this lowfrequency range with respect to the dynamics targets, are not directly taken into account for the construction of the reducedorder model. It should be noted that, with the large computational model used today, there are absolutely no difficulties to compute several thousands of elastic modes (global and local elastic modes) with a computational model having several millions of degrees of freedom. The problem which occurs is induced by the existence of an efficient sorting method which could be used to select the elastic modes as global elastic modes or as local elastic modes. In fact, for complex structures such as an automotive vehicle, it is very difficult to sort these elastic modes without an adapted algorithm. The reason why is mainly due to the fact that many elastic modes cannot clearly be identified as a global mode or as a local mode. In addition, although the reduced-order model must be constructed with respect to the global elastic modes, this reduced-order model must have the capability to predict the amplitudes of the responses of the structure in this low-frequency range. Since there are local elastic modes in the frequency band, a part of the mechanical energy is transferred from the global elastic modes to the local elastic modes which store this energy and then which induces an apparent damping at the resonances associated with the global elastic modes. This mechanism is exactly the phenomenon described and modeled by the fuzzy structure theory introduced in $[16,17]$ and for which a synthesis can be found in [6]; since 1993, much research has been published in this field [18-26].

The objective of this paper is double: (1) The first one is to develop a formulation and an algorithm which allow the 
"global elastic modes" and the "local elastic modes" to be separately calculated. (2) The second one is to construct a reducedorder model with the global elastic modes but in taking into account the effects of the local elastic modes as in the fuzzy structure theory, in order to correctly predict the frequency response functions in the low-frequency range.

It should be noted that our objective is not to exactly construct the "global" elastic modes and the "local" elastic modes as elements of the family of all the elastic modes, but to construct a projection basis made up of a family of "global elastic modes" (closed to the corresponding elastic modes which are dominated by global displacements) and of a family of "local elastic modes" (which are dominated by local displacements) such that the union of these two families constitutes a basis of the admissible space of displacements. Consequently, the computational model can be projected on the subspace spanned by this family in order to construct a reduced-order model for the frequency-response-functions calculation.

We have now to explain what will be the strategy for this double objective.

Concerning the first one, the main idea is to construct a spatial filtering of elastic modes such that the elastic modes having a wavelength less than a given characteristic value, be filtered. It can be seen that this notion is not really clear because the wavelength of an elastic mode for a complex heterogeneous structure does not exist (such a mode has a continuous spectrum of wavelength which is not reduced to a ray). There are very few studies, if any, for this area of structural dynamics. Spatial filtering of short wavelength achieved by regularization has been proposed in the context of the extraction of left eigenvectors from measured frequency response functions for experimental modal analysis [27] based on the use of a singular value decomposition to construct the spatial filtering scheme [28]. This interesting work is not adapted to our first objective for which a complete basis of the admissible space, made up of the two families (global and local elastic modes), has to be constructed with an algorithm which is not intrusive. The theory constructed and proposed in this paper is based on the introduction of a subspace of the admissible space for the calculation of the kinetic energy (but not for the elastic energy) which allows such a spatial filter to be constructed. A simple version of this approach consists in approximating the consistent mass matrix of the computational model by a lumped mass matrix allowing some local elastic modes to be filtered [29, 30]. When such a procedure of lumping mass is used, it is then assumed that the acceleration are approximatively constant in the neighborhood of the node in which the point mass is concentrated. Although properties of solutions obtained with lumped mass matrix has been studied (see for instance [31-33]), such a filter would depend on the mesh and the bandpass filter could not be selected. In addition, it would seem that no way exists to complete the construction of the basis including the local elastic modes (nevertheless, it should be recalled that the use of the lumped mass approximation coupled with the Guyan condensation [34] allows the first elastic modes to be calculated).

Concerning the second objective, the main problem which has to be solved is the selection of an approach which allows uncertainties in the computational model to be taken into account for the contribution of the local elastic modes in the frequency responses of the dynamical system. It is well known that the local elastic modes are not robust with respect to both the system parameters uncertainty and the model uncertainties induced by modeling errors. The parametric probabilistic approach is certainly the most efficient and effective method to take into account system parameters uncertainties (see for instance [35]) but does not have the capability to take into account model uncertainties. Unfortunately, the local elastic modes are very sensitive to model uncertainties. Recently, the nonparametric probabilistic approach [36,37] which has 
received several experimental validations (see for instance [26,38-41]) has been proposed as a possible way to take into account both the system parameters uncertainty and the model uncertainties. Consequently, such a probabilistic approach will be used to take into account uncertainties in the contribution of the local elastic modes.

The development begins with the introduction of the reference boundary value problem which has to be solved introducing a reduced-order model for the low-frequency range. A decomposition of the mass bilinear form adapted to the calculation of the low-frequency global modes in presence of numerous local modes is then presented. In a next section, a basis of the admissible space is introduced as the union of the families of the global and local elastic modes for which the methodology for their construction is given. The finite element approximation is then detailed for all the aspects of the method. In a last section devoted to the theory proposed, the nonparametric probabilistic model of uncertainties is introduced for the local elastic modes and, the random reduced matrix model and the random frequency response functions are constructed. The final part of the paper is devoted to an application which validates the theory proposed.

\section{Reference boundary value problem and its variational formulation}

In this section, we summarized very well known results for which details can be found, for instance, in [6]. The brief presentation given below allows the problem and notation to be defined.

Let $\Omega$ be the domain of a three dimensional linear damped structure which is fixed on the part $\Gamma_{0}$ of its boundary $\partial \Omega$ and free on the other part $\Gamma$. The outward unit normal of $\partial \Omega$ is denoted by $n$. We are interested in predicting the frequency response functions of this structure in the frequency band of analysis $\mathcal{B}=\left[\omega_{\min }, \omega_{\max }\right]$ with $0<\omega_{\min }$. Let $u(x, \omega)=\int_{-\infty}^{+\infty} e^{-i \omega t} u(x, t) d t$ be the Fourier transform of the displacement field $u=\left(u_{1}, u_{2}, u_{3}\right)$ for $\omega \in \mathcal{B}$, and where $x=\left(x_{1}, x_{2}, x_{3}\right)$ are the cartesian coordinates. The Fourier transform of the external prescribed volumetric and surface forces fields applied in $\Omega$ and on its boundary $\Gamma$ are denoted by $f_{v o l}(x, \omega)$ and $f_{\text {surf }}(x, \omega)$. The stress tensor $\sigma(x)$ is written as $\sigma_{j k}(x)=a_{j k \ell h}(x) \varepsilon_{\ell h}(u)+$ $i \omega b_{j k \ell h}(x) \varepsilon_{\ell h}(u)$ where $\varepsilon_{\ell h}(u)=\left(\partial u_{\ell} / \partial x_{h}+\partial u_{h} / \partial x_{\ell}\right) / 2$ is the linearized strain tensor. The fourth-order tensors $a(x)$ and $b(x)$ verify the usual properties of symmetry and positiveness. Then, the displacement field $u(\omega)$ verifies, for all $\omega \in \mathcal{B}$ and for $i=\{1,2,3\}$, the boundary value problem

$$
\begin{gathered}
-\omega^{2} \rho u_{i}-\partial \sigma_{i j} / \partial x_{j}=f_{\text {vol }, i} \text { in } \Omega, \\
\sigma_{i j} n_{j}=f_{\text {surf }, i} \text { on } \Gamma, \\
u=0 \text { on } \Gamma_{0},
\end{gathered}
$$

in which the mass density $x \mapsto \rho(x)$ is a positive and bounded function and where the convention for summations over repeated Latin indices is used.

Let $\mathbb{H}^{\text {comp }}$ be the complex space of all the square integrable functions from $\Omega$ into $\mathbb{C}^{3}$ equipped with the inner product $<u, v>=\int_{\Omega} u(x) \cdot \overline{v(x)} d x$ in which $u \cdot v=\sum_{j=1}^{3} u_{j} v_{j}$ and for which the associated norm is $\|u\|=<u, u>^{1 / 2}$. Let $\mathbb{V}^{\text {comp }}$ be 
the admissible function space for which $u=0$ on $\Gamma_{0}$ and which is a subspace of $\mathbb{H}^{\text {comp }}$. The subspaces of $\mathbb{H}^{\text {comp }}$ and $\mathbb{V}^{\text {comp }}$ made up of the $\mathbb{R}^{3}$-valued functions are denoted by $\mathbb{H}$ and $\mathbb{V}$.

Then, the variational formulation of the boundary value problem defined by Eq. (1) consists in finding the function $u(\omega)$ with values in $\mathbb{V}^{\text {comp }}$ such that, for all $v$ in $\mathbb{V}^{\text {comp }}$

$$
-\omega^{2} m(u, v)+i \omega d(u, v)+k(u, v)=g(v ; \omega)
$$

The mass sesquilinear form

$$
m(u, v)=\int_{\Omega} \rho(x) u(x) \cdot \overline{v(x)} d x
$$

defined on $\mathbb{H}^{\text {comp }} \times \mathbb{H}^{\text {comp }}$, is Hermitian and positive definite. The damping sesquilinear form $d(u, v)=\int_{\Omega} b_{j k \ell h}(x) \varepsilon_{\ell h}(u) \varepsilon_{j k}(\bar{v}) d x$ and the stiffness sesquilinear form $k(u, v)=\int_{\Omega} a_{j k \ell h}(x) \varepsilon_{\ell h}(u) \varepsilon_{j k}(\bar{v}) d x$, defined on $\mathbb{V}^{\text {comp }} \times \mathbb{V}^{\text {comp }}$, are Hermitian and positive definite. For all $\omega \in \mathcal{B}$, the antilinear form $g(v ; \omega)$ of the prescribed external forces is defined on $\mathbb{V}^{\operatorname{comp}}$ by $g(v ; \omega)=$ $\int_{\Gamma} f_{\text {surf }}(x, \omega) \cdot \overline{v(x)} d s(x)+\int_{\Omega} f_{v o l}(x, \omega) \cdot \overline{v(x)} d x$. The spectral problem associated with Eq. (2) consists in finding real $\lambda$ and $\varphi$ in $\mathbb{V}$ such that, for all $v$ in $\mathbb{V}$

$$
-\lambda m(\varphi, v)+k(\varphi, v)=0
$$

In this case, it is well known that there exists an increasing sequence of positive eigenvalues with finite multiplicity $0<$ $\lambda_{1} \leq \lambda_{2} \leq \ldots$. The elastic modes (the eigenfunctions) $\left\{\varphi_{\alpha}, \alpha \geq 1\right\}$ form a complete set in $\mathbb{V}$ which means that an arbitrary function $u$ belonging to $\mathbb{V}$ (or to $\mathbb{V}^{\text {comp }}$ ) can be expanded as

$$
u(x, \omega)=\sum_{\alpha=1}^{\infty} q_{\alpha}(\omega) \varphi_{\alpha}(x)
$$

in which $q_{\alpha}$ are the real (or the complex) generalized coordinates. The finite approximations at order $n$ is $u_{n}(x, \omega)=$ $\sum_{\alpha=1}^{n} q_{\alpha}(\omega) \varphi_{\alpha}(x)$ and allows the reduced-order model to be constructed. 


\section{Decomposition of the mass bilinear form adapted to the calculation of the low-frequency global modes in presence}

\section{of numerous local modes}

In this section, we introduce a decomposition of the mass bilinear form on $\mathbb{H} \times \mathbb{H}$ which is adapted to the calculation of the global elastic modes in the low-frequency band of analysis in which there are also a large number of local elastic modes. Therefore, we first introduce a projection operator on a finite dimension subspace of $\mathbb{H}$. Then, this operator is used to decompose the mass bilinear form into a reduced mass bilinear form and a complementary mass bilinear form.

\subsection{Decomposition of domain $\Omega$}

Domain $\Omega$ is partitioned into $n_{J}$ subdomains $\Omega_{j}^{\varepsilon}$ such that, for $j$ and $k$ in $\left\{1, \ldots, n_{J}\right\}$,

$$
\Omega=\bigcup_{j=1}^{n_{J}} \Omega_{j}^{\varepsilon} \quad, \quad \Omega_{j}^{\varepsilon} \cap \Omega_{k}^{\varepsilon}=\emptyset .
$$

The parameter $\varepsilon$ is the characteristic length of the subdomains. The choice of $\varepsilon$ is related to the highest "wavelength" of the global elastic modes that we want to extract in presence of numerous local modes. This parameter will be detailed in Section 4. Note that, for the finite element approximation, each subdomain $\Omega_{j}^{\varepsilon}$ of the partition of $\Omega$ will coincide with the union of some connected finite elements of the mesh of $\Omega$ (see Section 5).

\subsection{Projection operator}

Let $\mathbb{H}_{r}$ be the subspace of finite dimension $3 n_{J}$ of space $\mathbb{H}$ defined as the set all the functions which are constant on each $\Omega_{j}^{\varepsilon}$ for all $j$ in $\left\{1, \ldots, n_{J}\right\}$. Let $u \mapsto h_{\varepsilon}^{r}(u)$ be the linear operator from $\mathbb{H}$ into $\mathbb{H}_{r}$ defined by

$$
\left\{h_{\varepsilon}^{r}(u)\right\}(x)=\sum_{j=1}^{n_{J}} \mathbb{1}_{\Omega_{i}^{\varepsilon}}(x) \frac{1}{m_{j}} \int_{\Omega_{j}^{\varepsilon}} \rho\left(x^{\prime}\right) u\left(x^{\prime}\right) d x^{\prime},
$$

in which $x \mapsto \mathbb{1}_{\Omega_{j}^{\varepsilon}}(x)=1$ if $x$ is in $\Omega_{j}^{\varepsilon}$ and $=0$ otherwise. The local mass $m_{j}$ is defined, for all $j$ in $\left\{1, \ldots, n_{J}\right\}$, by

$$
m_{j}=\int_{\Omega_{j}^{\varepsilon}} \rho(x) d x
$$

Let $\mathbb{H}_{c}$ be the subspace of $\mathbb{H}$ constitutes of all the functions $u-h_{\varepsilon}^{r}(u)$ when $u$ runs through $\mathbb{H}$. Let $u \mapsto h_{\varepsilon}^{c}(u)$ be the linear operator from $\mathbb{H}$ into $\mathbb{H}_{c}$ defined by

$$
h_{\varepsilon}^{c}(u)=u-h_{\varepsilon}^{r}(u)
$$


It should be noted that $\mathbb{H}_{c}$ is then the image of linear operator $h_{\varepsilon}^{c}$ in $\mathbb{H}$. Function $h_{\varepsilon}^{r}(u)$ will also be denoted by $u^{r}$ and function $h_{\varepsilon}^{c}(u)$ by $u^{c}$. We then have $u=h_{\varepsilon}^{r}(u)+h_{\varepsilon}^{c}(u)$ that is to say, $u=u^{r}+u^{c}$. It can easily be verified that, for all $u$ in $\mathbb{H}$, we have $h_{\varepsilon}^{r}\left(h_{\varepsilon}^{r}(u)\right)=h_{\varepsilon}^{r}(u)$ and $h_{\varepsilon}^{c}\left(h_{\varepsilon}^{r}(u)\right)=h_{\varepsilon}^{r}\left(h_{\varepsilon}^{c}(u)\right)=0$. In addition, for $u=u^{r}+u^{c}$ in $\mathbb{H}$, we have $m\left(u^{r}, u^{c}\right)=0$ and consequently, $m\left((u+v)^{r},(u+v)^{c}\right)=0$ which yields $m\left(u^{r}, v^{c}\right)=0$ for all $u$ and $v$ in $\mathbb{H}$. It can then be deduced that linear operator $u \mapsto h_{\varepsilon}^{r}(u)$ is a projection operator and that $\mathbb{H}=\mathbb{H}_{r} \oplus \mathbb{H}_{c}$. To verify this last result, it must be proven that for all $u^{r}$ in $\mathbb{H}_{r}$ and for all $v^{c}$ in $\mathbb{H}_{c}$, the equality $u^{r}+v^{c}=0$ yields $u^{r}=0$ and $v^{c}=0$ that is the case because $m\left(u^{r}, v^{c}\right)=0$ and the bilinear form $m$ is positive definite.

\subsection{Reduced and complementary mass bilinear forms}

For all $u$ and $v$ in $\mathbb{H}$, the reduced mass bilinear form is defined by

$$
m^{r}(u, v)=m\left(h_{\varepsilon}^{r}(u), v\right)=m\left(u, h_{\varepsilon}^{r}(v)\right)
$$

and the complementary mass bilinear form is defined by

$$
m^{c}(u, v)=m\left(h_{\varepsilon}^{c}(u), v\right)=m\left(u, h_{\varepsilon}^{c}(v)\right)
$$

From $u=h_{\varepsilon}^{r}(u)+h_{\varepsilon}^{c}(u)$, it can be deduced that

$$
m(u, v)=m^{r}(u, v)+m^{c}(u, v)
$$

(i) Kernels of the operators associated with the bilinear forms $m^{c}$ and $m^{r}$. The kernel of the operator associated with the bilinear form $m^{c}$ is made up of the solutions $u$ of $m^{c}(u, v)=0$ for all $v$ and consequently, coincides with the kernel of linear operator $u \mapsto h_{\varepsilon}^{c}(u)=u-h_{\varepsilon}^{r}(u)$, that is to say coincides with the solutions of $u-h_{\varepsilon}^{r}(u)=0$ which is then a subset of $\mathbb{H}_{r}$. It can be verified that $\mathbb{H}_{r}$ is also a subset of this kernel and therefore, this kernel is $\mathbb{H}_{r}$. Similarly, the kernel of the operator associated with the bilinear form $m^{r}$ is made up of the solutions $u$ of $m^{r}(u, v)=0$ for all $v$ and consequently, coincides with the kernel of linear operator $u \mapsto h_{\varepsilon}^{r}(u)$. Since $h_{\varepsilon}^{r}\left(h_{\varepsilon}^{c}(u)\right)=0$, we conclude that $\mathbb{H}_{c}$ is a subset of this kernel. It can be verified that this kernel is also a subset of $\mathbb{H}_{c}$ and therefore, this kernel is $\mathbb{H}_{c}$.

(ii) Positiveness. Let $u^{r}$ be a function of $\mathbb{H}_{r} \subset \mathbb{H}$ and $u^{c}$ be a function of $\mathbb{H}_{c} \subset \mathbb{H}$. Since $h_{\varepsilon}^{c}\left(u^{r}\right)=h_{\varepsilon}^{r}\left(u^{c}\right)=0$, it can be deduced that, for all $v$ in $\mathbb{H}, m^{r}\left(u^{c}, v\right)=m^{c}\left(u^{r}, v\right)=0$. Using Eqs. (10) and (11), it can easily be proven that the bilinear forms $m^{r}(u, v)$ and $m^{c}(u, v)$ are symmetric on $\mathbb{H} \times \mathbb{H}$. For all $u=u^{r}+u^{c}$ in $\mathbb{H}$, since $m\left(u^{r}, u^{c}\right)=m\left(u^{c}, u^{r}\right)=0$ and since $m(u, v)$ is positive definite on $\mathbb{H} \times \mathbb{H}$, it can be deduced that $m^{r}(u, u) \geq 0$ and $m^{c}(u, u) \geq 0$ which means that $m^{r}(u, v)$ and 
$m^{c}(u, v)$ are positive semi-definite on $\mathbb{H} \times \mathbb{H}$.

(iii) Mass conservation. Let 1 be the function in $\mathbb{H}$ such that $1(x)=(1,1,1)$. The total mass $M$ of the dynamical system can be calculated by $M=m(1,1) / 3$. Similarly, the total mass $M^{r}$ associated with the reduced mass bilinear form and the total mass $M^{c}$ associated with the complementary mass bilinear form are defined by $M^{r}=m^{r}(1,1) / 3$ and $M^{c}=m^{c}(1,1) / 3$. Since $h_{\varepsilon}^{r}(1)=1$, it can be deduced that $M=M^{r}$ and since $m^{c}(1,1)=m(1,1)-m^{r}(1,1)$, that $M^{c}=M-M^{r}=0$.

\section{Global and local elastic modes as a basis of the admissible space}

The reduced mass bilinear form can be rewritten as

$$
\begin{aligned}
m^{r}(u, v) & =\int_{\Omega} \rho(x)\left\{\sum_{j=1}^{n_{J}} \mathbb{1}_{\Omega_{j}^{\varepsilon}}(x) \frac{1}{m_{j}} \int_{\Omega_{j}^{\varepsilon}} \rho\left(x^{\prime}\right) u\left(x^{\prime}\right) d x^{\prime}\right\} \cdot v(x) d x \\
& =\sum_{j=1}^{n_{J}} m_{j}\left\{\frac{1}{m_{j}} \int_{\Omega_{j}^{\varepsilon}} \rho(x) u(x) d x\right\} \cdot\left\{\frac{1}{m_{j}} \int_{\Omega_{j}^{\varepsilon}} \rho(x) v(x) d x\right\} \\
& =\sum_{j=1}^{n_{J}} m_{j} f_{j}(u) \cdot f_{j}(v),
\end{aligned}
$$

in which $f_{j}(u)=\left(1 / m_{j}\right) \int_{\Omega_{j}^{\varepsilon}} \rho(x) u(x) d x$. From Eq. (13), it can be deduced that the reduced mass bilinear form can be interpreted as the mass bilinear form of a discrete system made up of $n_{J}$ masses $m_{j}$ affected to the degrees of freedom defined by $f_{j}(u)$. Then, by replacing $m(u, v)$ by $m^{r}(u, v)$ in the spectral problem defined by Eq. (4), all the "wavelengths" of the elastic modes of this new spectral problem will be greater than the parameter $\varepsilon$ defining the size of the subdomains $\Omega_{j}{ }_{j}$. Thus, only the global elastic modes will remain.

\subsection{Global elastic modes}

The global elastic modes are then defined as the eigenfunctions $\varphi^{g}$ in $\mathbb{V}$ associated with the eigenvalues $\lambda^{g}$, verifying, for all $v$ in $\mathbb{V}$, the following global spectral problem,

$$
-\lambda^{g} m^{r}\left(\varphi^{g}, v\right)+k\left(\varphi^{g}, v\right)=0,
$$

which can be rewritten as $-\mu^{g} k\left(\varphi^{g}, v\right)+m^{r}\left(\varphi^{g}, v\right)=0$ in which $\mu^{g}=1 / \lambda^{g}$. The subspace of $\mathbb{H}$ associated with $\mu_{0}^{g}=0$ is spanned by the solutions in $u$ of $m^{r}(u, v)=0$ for all $v$ and consequently, is the space $\mathbb{H}_{c}$ which is not a subset of $\mathbb{V}$. We then search for the solutions, functions $\varphi^{g}$ in the subspace $\mathbb{V} \cap\left\{\mathbb{H} \backslash \mathbb{H}_{c}\right\}$ whose dimension is finite and equal to $3 n_{J}$, associated with the positive eigenvalues $\mu^{g}>0$. This generalized eigenvalue problem in $\mu^{g}$ then admits a decreasing finite sequence of positive eigenvalues $\mu_{1}^{g} \geq \ldots \geq \mu_{3 n_{J}}^{g}>0$, corresponding to the increasing family $0<\lambda_{1}^{g} \leq \ldots \leq \lambda_{3 n_{J}}^{g}$, associated with the finite family of algebraically independent functions $\left\{\varphi_{1}^{g}, \ldots, \varphi_{3 n_{J}}^{g}\right\}$ made up of functions in $\mathbb{V}$ and which satisfies the 
following orthogonality properties,

$$
k\left(\varphi_{\alpha}^{g}, \varphi_{\beta}^{g}\right)=\lambda_{\alpha}^{g} \delta_{\alpha \beta} \quad, \quad m^{r}\left(\varphi_{\alpha}^{g}, \varphi_{\beta}^{g}\right)=\delta_{\alpha \beta}
$$

and for all $\alpha=1, \ldots, 3 n_{J}$ and for all $v^{c}$ in $\mathbb{H}_{c}$, we have

$$
m^{r}\left(\varphi_{\alpha}^{g}, v^{c}\right)=0
$$

The family $\left\{\varphi_{1}^{g}, \ldots, \varphi_{3 n_{J}}^{g}\right\}$ is defined as the family of the global elastic modes although these functions are not, in general, some elements of the family of the elastic modes, but represent some approximations of such elements.

\subsection{Local elastic modes}

The local elastic modes are then defined as the eigenfunctions $\varphi^{\ell}$ in $\mathbb{V}$ associated with the eigenvalues $\lambda^{\ell}$, verifying, for all $v$ in $\mathbb{V}$, the following global spectral problem,

$$
-\lambda^{\ell} m^{c}\left(\varphi^{\ell}, v\right)+k\left(\varphi^{\ell}, v\right)=0
$$

which can be rewritten as $-\mu^{\ell} k\left(\varphi^{\ell}, v\right)+m^{c}\left(\varphi^{\ell}, v\right)=0$ in which $\mu^{\ell}=1 / \lambda^{\ell}$. The subspace of $\mathbb{H}$ associated with $\mu_{0}^{\ell}=0$ is spanned by the solutions in $u$ of $m^{c}(u, v)=0$ for all $v$ and consequently, is the space $\mathbb{H}_{r}$ for which $\mathbb{H}_{r} \cap \mathbb{V}$ is the empty set. We then search for the solutions, functions $\varphi^{\ell}$ in the infinite dimension subspace $\mathbb{V} \cap\left\{\mathbb{H} \backslash \mathbb{H}_{r}\right\}$, associated with the positive eigenvalues $\mu^{\ell}>0$. This generalized eigenvalue problem in $\mu^{\ell}$ then admits a decreasing sequence of positive eigenvalues $\mu_{1}^{\ell} \geq \mu_{2}^{\ell} \geq \ldots>0$, corresponding to the increasing family $0<\lambda_{1}^{\ell} \leq \lambda_{2}^{\ell} \leq \ldots$, associated with the infinite family of functions $\left\{\varphi_{1}^{\ell}, \varphi_{2}^{\ell}, \ldots,\right\}$ made up of functions in $\mathbb{V}$ and which satisfies the following orthogonality properties,

$$
k\left(\varphi_{\alpha}^{\ell}, \varphi_{\beta}^{\ell}\right)=\lambda_{\alpha}^{\ell} \delta_{\alpha \beta} \quad, \quad m^{c}\left(\varphi_{\alpha}^{\ell}, \varphi_{\beta}^{\ell}\right)=\delta_{\alpha \beta},
$$

and for all $\alpha=1,2, \ldots$, and for all $v^{r}$ in $\mathbb{H}_{r}$, we have

$$
m^{c}\left(\varphi_{\alpha}^{\ell}, v^{r}\right)=0
$$


The family $\left\{\varphi_{1}^{\ell}, \varphi_{2}^{\ell}, \ldots,\right\}$ is defined as the family of the local elastic modes although these functions are not, in general, some elements of the family of the elastic modes, but represent some approximations of such elements.

\subsection{Basis of the admissible space}

It is proven in Appendix A that the family $\left\{\varphi_{1}^{g}, \ldots, \varphi_{3 n_{J}}^{g}, \varphi_{1}^{\ell}, \varphi_{2}^{\ell}, \ldots\right\}$ is a basis of space $\mathbb{V}$ and consequently, all $u(\omega)$ in $\mathbb{V}^{\text {comp }}$ can be written as

$$
u(\omega)=\sum_{\alpha=1}^{3 n_{J}} q_{\alpha}^{g}(\omega) \varphi_{\alpha}^{g}+\sum_{\alpha^{\prime}=1}^{+\infty} q_{\alpha^{\prime}}^{\ell}(\omega) \varphi_{\alpha^{\prime}}^{\ell}
$$

in which $q_{\alpha}^{g}(\omega)$ and $q_{\alpha^{\prime}}^{\ell}(\omega)$ are complex numbers.

\section{Finite element approximation}

\subsection{Finite Element model of the mean boundary value problem}

The finite element method is applied to construct a finite approximation of Eq. (2). The complex vector $\mathbb{U}(\omega)$ of the $m$ DOF satisfies the complex matrix equation,

$$
\left(-\omega^{2}[\mathbb{M}]+i \omega[\mathbb{D}]+[\mathbb{K}]\right) \mathbb{U}(\omega)=\mathbb{F}(\omega)
$$

in which $[\mathbb{M}],[\mathbb{D}]$ and $[\mathbb{K}]$ are respectively the $(m \times m)$ positive-definite symmetric real mass, damping and stiffness matrices and where $\mathbb{F}(\omega)$ is relative to the discretization of the external forces. The finite element discretization of the spectral problem defined by Eq. (4) consists in finding $\lambda$ and $\phi$ in $\mathbb{R}^{m}$ such that

$$
[\mathbb{K}] \phi=\lambda[\mathbb{M}] \phi
$$

Then, the approximation $\mathbb{U}_{n}(\omega)$ at order $n$ of $\mathbb{U}(\omega)$ is written as

$$
\mathbb{U}_{n}(\omega)=\sum_{\alpha=1}^{n} q_{\alpha}(\omega) \phi_{\alpha}=[\Phi] q
$$

in which $q=\left(q_{1}, \ldots, q_{n}\right)$ is the complex vector of the $n$ generalized coordinates and where $[\Phi]=\left[\phi_{1} \ldots \phi_{n}\right]$ is the $(m \times n)$ real matrix of the elastic modes associated with the $n$ first eigenvalues.

The finite element discretization $\mathbb{U}$ of $u$ can be written as $\mathbb{U}=\mathbb{U}^{r}+\mathbb{U}^{c}$, in which $\mathbb{U}^{r}=\left[H_{\varepsilon}^{r}\right] \mathbb{U}$ and $\mathbb{U}^{c}=\left[H_{\varepsilon}^{c}\right] \mathbb{U}=\mathbb{U}-\mathbb{U}^{r}$ 
which shows that $\left[H_{\varepsilon}^{c}\right]=\left[I_{m}\right]-\left[H_{\varepsilon}^{r}\right]$. The $(m \times m)$ matrix $\left[H_{\varepsilon}^{r}\right]$ is relative to the finite element discretization of the projection operator $h_{\varepsilon}^{r}$ defined by Eq. (7).

\subsection{Global and local elastic modes}

Two methods are proposed to calculate the global and the local elastic modes that will be used to reduce the matrix equation.

\subsubsection{Direct method}

In such a method, the global and the local elastic modes are directly calculated using the decomposition of the mass matrix $[\mathbb{M}]$ relative to the decomposition of the mass bilinear form defined by Eq. (12). The finite element discretization of the spectral problems defined by Eqs. (14) and (17) yields, for the global elastic modes $\phi^{g}$ in $\mathbb{R}^{m}$, the generalized eigenvalue problem

$$
[\mathbb{K}] \phi^{g}=\lambda^{g}\left[\mathbb{M}^{r}\right] \phi^{g}
$$

and, for the local elastic modes $\phi^{\ell}$ in $\mathbb{R}^{m}$, the generalized eigenvalue problem

$$
[\mathbb{K}] \phi^{\ell}=\lambda^{\ell}\left[\mathbb{M}^{c}\right] \phi^{\ell}
$$

where the $(m \times m)$ matrices $\left[\mathbb{M}^{r}\right]$ and $\left[\mathbb{M}^{c}\right]$ are relative to the finite element discretization of the bilinear forms $m^{r}(u, v)$ and $m^{c}(u, v)$ defined by Eqs. (10) and (11). The matrix $\left[\mathbb{M}^{r}\right]$ is constructed such that $\left[\mathbb{M}^{r}\right]=[\mathbb{M}]\left[H_{\varepsilon}^{r}\right]=\left[H_{\varepsilon}^{r}\right]^{T}[\mathbb{M}]=\left[H_{\varepsilon}^{r}\right]^{T}[\mathbb{M}]\left[H_{\varepsilon}^{r}\right]$, and using Eq. (12) yields $\left[\mathbb{M}^{c}\right]=[\mathbb{M}]-\left[\mathbb{M}^{r}\right]$. The matrices $\left[\mathbb{M}^{r}\right]$ and $\left[\mathbb{M}^{c}\right]$ are symmetric and positive but are not positive definite.

\subsubsection{Double projection method}

This method is less intrusive with respect to the commercial software and less time-consuming than the direct method. The solutions of the generalized eigenvalue problems defined by Eqs. (24) and (25) are then written, for $n$ sufficiently large, as

$$
\phi^{g}=[\Phi] \widetilde{\phi}^{g} \quad, \quad \phi^{\ell}=[\Phi] \widetilde{\phi}^{\ell} .
$$


The generalized global elastic modes are the solutions of the generalized eigenvalue problem

$$
[\widetilde{K}] \widetilde{\phi}^{g}=\lambda^{g}\left[\widetilde{M}^{r}\right] \widetilde{\phi}^{g}
$$

in which $\left[\widetilde{M}^{r}\right]=\left[\Phi_{\varepsilon}^{r}\right]^{T}[\mathbb{M}]\left[\Phi_{\varepsilon}^{r}\right]$ and $[\widetilde{K}]=[\Phi]^{T}[\mathbb{K}][\Phi]$, and where the $(m \times n)$ real matrix $\left[\Phi_{\varepsilon}^{r}\right]$ is such that $\left[\Phi_{\varepsilon}^{r}\right]=\left[H_{\varepsilon}^{r}\right][\Phi]$. The generalized local elastic modes are the solutions of the generalized eigenvalue problem

$$
[\widetilde{K}] \widetilde{\phi}^{\ell}=\lambda^{\ell}\left[\widetilde{M}^{c}\right] \widetilde{\phi}^{\ell}
$$

in which $\left[\widetilde{M}^{c}\right]=\left[\Phi_{\varepsilon}^{c}\right]^{T}[\mathbb{M}]\left[\Phi_{\varepsilon}^{c}\right]$ and where the $(m \times n)$ real matrix $\left[\Phi_{\varepsilon}^{c}\right]$ is such that $\left[\Phi_{\varepsilon}^{c}\right]=\left[H_{\varepsilon}^{c}\right][\Phi]=[\Phi]-\left[\Phi_{\varepsilon}^{r}\right]$.

\subsection{Mean reduced matrix model}

The mean reduced matrix model is obtained using the projection of $\mathbb{U}(\omega)$ on the subspace of $\mathbb{C}^{m}$ spanned by the family $\left\{\phi_{1}^{g}, \ldots, \phi_{n_{g}}^{g}, \phi_{1}^{\ell}, \ldots, \phi_{n_{\ell}}^{\ell}\right\}$ of real vectors associated with the $n_{g}$ first global elastic modes such that $n_{g} \leq 3 n_{J} \leq m$ and with the $n_{\ell}$ first local elastic modes such that $n_{\ell} \leq m$. It should be noted that, if the double projection method is used, then we must have $n_{g} \leq n, n_{\ell} \leq n$ and $n_{t} \leq n$ in which $n_{t}=n_{g}+n_{\ell}$. Then, the approximation $\mathbb{U}_{n_{g}, n_{\ell}}(\omega)$ of $\mathbb{U}(\omega)$ at order $\left(n_{g}, n_{\ell}\right)$ is written as

$$
\mathbb{U}_{n_{g}, n_{\ell}}(\omega)=\sum_{\alpha=1}^{n_{g}} q_{\alpha}^{g}(\omega) \phi_{\alpha}^{g}+\sum_{\beta=1}^{n_{\ell}} q_{\beta}^{\ell}(\omega) \phi_{\beta}^{\ell}
$$

Let $q(\omega)=\left(q^{g}(\omega), q^{\ell}(\omega)\right)$ be the vector in $\mathbb{C}^{n}$ of all the generalized coordinates such that $q^{g}(\omega)=\left(q_{1}^{g}(\omega), \ldots, q_{n_{g}}^{g}(\omega)\right)$ and $q^{\ell}(\omega)=\left(q_{1}^{\ell}(\omega), \ldots, q_{n_{\ell}}^{\ell}(\omega)\right)$. Consequently, vector $q(\omega)$ is solution of the following mean reduced matrix equation such that

$$
\left(-\omega^{2}[M]+i \omega[D]+[K]\right) q(\omega)=\mathcal{F}(\omega)
$$

where $[M],[D]$ and $[K]$ are the $\left(n_{t} \times n_{t}\right)$ mean generalized mass, damping and stiffness matrices defined by blocks as

$$
[M]=\left[\begin{array}{cc}
M^{g g} & M^{g \ell} \\
\left(M^{g \ell}\right)^{T} & M^{\ell \ell}
\end{array}\right],[D]=\left[\begin{array}{cc}
D^{g g} & D^{g \ell} \\
\left(D^{g \ell}\right)^{T} & D^{\ell \ell}
\end{array}\right],[K]=\left[\begin{array}{cc}
K^{g g} & K^{g \ell} \\
\left(K^{g \ell}\right)^{T} & K^{\ell \ell}
\end{array}\right] .
$$


Let $A$ (or $\mathbb{A}$ ) be denoting $M, D$ or $K$ (or $\mathbb{M}, \mathbb{D}$ or $\mathbb{K}$ ). Therefore, the block matrices are defined by

$$
[A]_{\alpha \beta}^{g g}=\left(\phi_{\alpha}^{g}\right)^{T}[\mathbb{A}] \phi_{\beta}^{g},[A]_{\alpha \beta}^{g \ell}=\left(\phi_{\alpha}^{g}\right)^{T}[\mathbb{A}] \phi_{\beta}^{\ell},[A]_{\alpha \beta}^{\ell \ell}=\left(\phi_{\alpha}^{\ell}\right)^{T}[\mathbb{A}] \phi_{\beta}^{\ell}
$$

The matrices $[K]^{g g}$ and $[K]^{\ell \ell}$ are diagonal. The generalized force is a vector in $\mathbb{C}^{n_{t}}$ which is written as $\mathcal{F}(\omega)=\left(\mathcal{F}^{g}(\omega), \mathcal{F}^{\ell}(\omega)\right)$ in which

$$
\mathcal{F}_{\alpha}^{g}(\omega)=\left(\phi_{\alpha}^{g}\right)^{T} \mathbb{F}(\omega) \quad, \quad \mathcal{F}_{\alpha}^{\ell}(\omega)=\left(\phi_{\alpha}^{\ell}\right)^{T} \mathbb{F}(\omega)
$$

The eigenvalues $\lambda^{g}$ of the global spectral problem and the eigenvalues $\lambda^{\ell}$ of the local spectral problem are strictly positive. Consequently, the mean generalized mass, damping and stiffness matrices are positive definite. Then, for all $\omega$ fixed in $\mathcal{B}$, the generalized coordinates are calculated by inverting Eq. (30) and the response $\mathbb{U}_{n_{g}, n_{\ell}}(\omega)$ is calculated using Eq. (29).

\section{Probabilistic model of uncertainties for the local elastic modes, random reduced matrix model and random fre- quency response functions}

In the low-frequency range, the first global elastic modes are not really sensitive to uncertainties introduced in the computational model. Nevertheless, we have assumed that the structure under consideration had also local elastic modes in the same low-frequency band. It is well known that the modal density of such local modes increases rapidly with the frequency and that, in addition, the local modes are sensitive both to the system parameters uncertainties and to the model errors which induce model uncertainties. In order to improve the predictability of the computational model, the nonparametric probabilistic approach (see $[36,37])$ is used to take into account uncertainties for the local-elastic-modes contribution.

\subsection{Random reduced matrix model}

The nonparametric probabilistic approach consists in replacing the matrices of the reduced mean matrix model by random matrices for which the probability distributions are constructed by using the maximum entropy principle [42,43] with the constraints defined by the available information. Then, the random generalized mass, damping and stiffness matrices are written as

$$
[M]=\left[\begin{array}{cc}
M^{g g} & M^{g \ell} \\
\left(M^{g \ell}\right)^{T} & M^{\ell \ell}
\end{array}\right],[D]=\left[\begin{array}{cc}
D^{g g} & D^{g \ell} \\
\left(D^{g \ell}\right)^{T} & D^{\ell \ell}
\end{array}\right],[K]=\left[\begin{array}{cc}
K^{g g} & K^{g \ell} \\
\left(K^{g \ell}\right)^{T} & K^{\ell \ell}
\end{array}\right],
$$


in which the random matrices $\left[M^{\ell \ell}\right],\left[D^{\ell \ell}\right]$ and $\left[K^{\ell \ell}\right]$ are with values in the set of all the positive-definite symmetric $\left(n_{\ell} \times n_{\ell}\right)$ real matrices, for which their mean values are such that $E\left\{\left[M^{\ell \ell}\right]\right\}=\left[M^{\ell \ell}\right], E\left\{\left[D^{\ell \ell}\right]\right\}=\left[D^{\ell \ell}\right]$ and $E\left\{\left[K^{\ell \ell}\right]\right\}=\left[K^{\ell \ell}\right]$, and finally, verify the following inequalities $E\left\{\left\|\left[M^{\ell}\right]^{-1}\right\|_{F}^{2}\right\}<+\infty, E\left\{\left\|\left[D^{\ell \ell}\right]^{-1}\right\|_{F}^{2}\right\}<+\infty$ and $E\left\{\left\|\left[K^{\ell \ell}\right]^{-1}\right\|_{F}^{2}\right\}<+\infty$ which assure that there exists a second-order random solution to the stochastic reduced-order equation (in the above equations, $E$ is the mathematical expectation and $\|A\|_{F}^{2}=\sum_{j=1}^{n_{\ell}} \sum_{k=1}^{n_{\ell}}[A]_{j k}^{2}$ ). Under the constraints defined by the above available information, the use of the maximum entropy principle shows that these three random matrices are statistically independent and allows the probability distribution of each random matrix $\left[M^{\ell \ell}\right],\left[D^{\ell \ell}\right]$ or $\left[K^{\ell \ell}\right]$ to be explicitly constructed. The probability distribution of each random matrix $\left[M^{\ell \ell}\right],\left[D^{\ell \ell}\right]$ or $\left[K^{\ell \ell}\right]$ depend on the mean value $\left[M^{\ell \ell}\right],\left[D^{\ell \ell}\right]$ or $\left[K^{\ell \ell}\right]$ and on a dispersion parameter $\delta_{M}$, $\delta_{D}$ or $\delta_{K}$ defined by

$$
\delta_{A}^{2}=\frac{E\left\{\left\|\left[A^{\ell \ell}\right]-\left[A^{\ell \ell}\right]\right\|_{F}^{2}\right\}}{\left\|\left[A^{\ell \ell}\right]\right\|_{F}^{2}},
$$

in which $A$ (or $A$ ) is $M, D$ or $K$ (or, $M, D$ or $K$ ). The dispersion parameters allow the level of uncertainties to be controlled. In addition, it can be found in $[36,37]$ an algebraic representation of such a random matrix which allows independent realizations to be explicitly constructed useful to solve the random equations by the Monte Carlo method. For each random matrix, this random generator only depends on the dimension of the matrix, on its mean value and on the dispersion parameter.

\subsection{Random frequency responses}

The random response $U_{n_{g}, n_{l}}(\omega)$ is then written as

$$
U_{n_{g}, n_{\ell}}(\omega)=\sum_{\alpha=1}^{n_{g}} Q_{\alpha}^{g}(\omega) \phi_{\alpha}^{g}+\sum_{\beta=1}^{n_{\ell}} Q_{\beta}^{\ell}(\omega) \phi_{\beta}^{\ell}
$$

in which the random vector $Q(\omega)=\left(Q^{g}(\omega), Q^{\ell}(\omega)\right)$ with valued in $\mathbb{C}^{n_{t}}$ of all the generalized coordinates is such that $Q^{g}(\omega)=\left(Q_{1}^{g}(\omega), \ldots, Q_{n_{g}}^{g}(\omega)\right)$ and $Q^{\ell}(\omega)=\left(Q_{1}^{\ell}(\omega), \ldots, Q_{n_{\ell}}^{\ell}(\omega)\right)$. Consequently, vector $Q(\omega)$ is solution of the following stochastic reduced matrix equation such that

$$
\left(-\omega^{2}[M]+i \omega[D]+[K]\right) Q(\omega)=\mathcal{F}(\omega) .
$$

This equation is solved using the Monte Carlo simulation method. 


\subsection{Remark concerning the connection with the fuzzy structure theory}

Let us consider a heterogeneous structure with a main part (the master structure) made up of a stiff structure and with secondary parts (the fuzzy substructure) made up of flexible substructures attached to the master structure. We can now see the connection of the above development with the fuzzy structure theory for which the global elastic modes are related to the "master structure" and the local elastic modes are related to the "fuzzy structure" corresponding to the hidden dynamical DOF associated with the local elastic modes. Let us assume that $\mathcal{F}^{\ell}(\omega)$ is zero (the external forces are only applied to the master structure (the stiff part). Eliminating $Q^{\ell}(\omega)$ in Eq. (37) yields

$$
-i \omega\left(\left[Z^{g g}(\omega)\right]+[Z(\omega)]\right) Q^{g}(\omega)=\mathcal{F}^{g}(\omega)
$$

in which $\left[Z^{g g}(\omega)\right]$ is the reduced impedance matrix of the master structure which is such that $-i \omega\left[Z^{g g}(\omega)\right]=-\omega^{2}\left[M^{g g}\right]+$ $i \omega\left[D^{g g}\right]+\left[K^{g g}\right]$. The matrix $[Z(\omega)]$ is the reduced impedance random matrix of the fuzzy substructure which can be written as $i \omega[Z(\omega)]=-\omega^{2}[R(\omega)]+i \omega[I(\omega)]$ in which the symmetric real random matrix $[R(\omega)]$ corresponds to the conservative effects of the fuzzy substructure on the master structure and where the real symmetric positive random matrix $[I(\omega)]$ corresponds to the additional damping induced by the fuzzy substructure in the master structure, due to the the mechanical energy stored by the local elastic modes of the fuzzy substructure. With such a framework of the fuzzy structure theory, the DOF in the fuzzy substructure are not observed and consequently, the vector of DOF $\left\{U_{n_{g}}(\omega)\right\}_{\text {mast }}$ in the master structure (the stiff part) is written as $\left\{U_{n_{g}}(\omega)\right\}_{\text {mast }} \simeq \sum_{\alpha=1}^{n_{g}} Q_{\alpha}^{g}(\omega)\left\{\phi_{\alpha}^{g}\right\}_{\text {mast }}$.

\section{Application and validation}

\subsection{Mean Finite Element Model}

The dynamical system is made up of 12 flexible panels and of a stiff structure (see Fig. 1). Each panel is a rectangular, homogeneous, isotropic, thin plate with constant thickness $0.002 \mathrm{~m}$, width $4.0 \mathrm{~m}$, length $4.0 \mathrm{~m}$, mass density $7,800 \mathrm{~kg} / \mathrm{m}^{3}$, Poisson ratio 0.29 . The Young modulus of the 12 panels are respectively, $1.31 \times 10^{12}, 1.47 \times 10^{12}, 1.54 \times 10^{12}, 1.74 \times 10^{12}$, $1.47 \times 10^{12}, 1.71 \times 10^{12}, 0.49 \times 10^{12}, 1.09 \times 10^{12}, 1.74 \times 10^{12}, 1.34 \times 10^{12}, 1.68 \times 10^{12}$ and $0.61 \times 10^{12} \mathrm{~N} / \mathrm{m}^{2}$. The rigid structure is composed of rectangular, homogeneous, isotropic, thin plates with a constant thickness $0.017 \mathrm{~m}$, width $2.0 \mathrm{~m}$, mass density $9,800 \mathrm{~kg} / \mathrm{m}^{3}$, Poisson ratio 0.29 , Young's modulus $2.1 \times 10^{12} \mathrm{~N} / \mathrm{m}^{2}$. The coordinate system $($ Oxyx $)$ is shown in Fig. 1. The four nodes corresponding to the four corners $(0,0,0),(26,0,0),(26,0,20)$ and $(0,0,20)$ are fixed. The frequency band of analysis is $\mathcal{B}=2 \pi \times] 0,11] \mathrm{rad} / \mathrm{s}$. The finite element model is made up of 64 Kirchhoff plate elements for each panel and 456 Kirchhoff plate elements for the rigid structure. The structure has $m=13,014$ DOF.

\subsection{Modal analysis, global and local elastic modes}

In a first step, the elastic modes are calculated with the finite element model defined by Eq. (22). There are 86 eigenfrequencies in the frequency band of analysis $\mathcal{B}$ and $n=120$ eigenfrequencies in the band $] 0,13.2] \mathrm{Hz}$. The first elastic 
mode $\phi_{1}$ and the second elastic mode $\phi_{2}$ are displayed in Fig. 2 and Fig. 3 which show that $\phi_{1}$ is a local elastic mode while $\phi_{2}$ is a global elastic mode with an important local displacement. In a second step, the global and local elastic modes are constructed using the double projection method introduced in Section 5.3. The matrix $\left[H_{\varepsilon}^{r}\right]$ relative to the discretization of the operator $h_{\varepsilon}^{r}$ is constructed for $\varepsilon=3.0 \mathrm{~m}$. This value of $\varepsilon$ results from a parametric analysis for which calculations have been carried our for the following increasing values 1.5, 2, 2.5 and 3 of $\varepsilon$ until the separation between the two families of global and local elastic modes be obtained. In band $] 0,13.2] \mathrm{Hz}$, there are $n_{g}=8$ global elastic modes and $n_{\ell}=112$ local elastic modes. Fig. 4 displays the distribution of the number of eigenfrequencies for the global elastic modes and for the local elastic modes. It can be seen that there are numerous local modes intertwined with the global elastic modes. The four first global elastic modes $\phi_{1}^{g}, \phi_{2}^{g}, \phi_{3}^{g}$ and $\phi_{4}^{g}$ are shown in Fig. 5 to Fig. 8.

\subsection{Frequency responses calculated with the mean model}

For all $\omega \in \mathcal{B}$, the structure is subjected to an external point load equal to $1 \mathrm{~N}$ applied to the node whose coordinates are $(10,0,7)$ located in the stiff part. The mean damping matrix is constructed using a Rayleigh model corresponding to a damping rate $\xi=0.04$ for the eigenfrequency $f_{1}=1.67 \mathrm{~Hz}$ and for the eigenfrequency $f_{120}=13.2 \mathrm{~Hz}$. The response is calculated at two observation points, the point Pobs 1 located in the stiff part at the node whose coordinates are $(19,0,7)$ and the point Pobs 2 located in the flexible part at the node whose coordinates are $(10,0,10)$ (see Fig. 1). The response is calculated for different projections associated with the different bases: for the initial elastic modes with Eq. (23) ( $n=120)$, for global elastic modes with Eq. (29) $\left(n_{g}=8\right.$ and $\left.n_{\ell}=0\right)$, for local elastic modes with Eq. (29) $\left(n_{g}=0\right.$ and $\left.n_{\ell}=112\right)$ and finally, for global and local elastic modes with Eq. (29) $\left(n_{g}=8\right.$ and $\left.n_{\ell}=112\right)$. The modulus in log scale of the responses are displayed in Fig. 9 and Fig. 10. It can be seen that the responses calculated using global and local elastic modes are exactly the same that the response calculated using the initial elastic modes. For point Pobs ${ }_{1}$ in the stiff part, the contribution of the global elastic modes is preponderant in the very low-frequency band but the contribution of the local elastic modes becomes not negligible in the high part of the low-frequency band. For point Pobs 2 in the flexible part, the contribution of the local elastic modes is important except for the first resonance corresponding to the first global elastic mode (because the flexible plates follow the stiff part in its displacement).

\subsection{Random frequency responses calculated with the stochastic model}

The nonparametric probabilistic approach is used for the contribution of the local elastic modes as explained in Section 6. The dispersion parameters (which should be identified experimentally or which can be used as superparameters for a sensitivity analysis) are presently chosen as $\delta_{M}=0.1, \delta_{D}=0.1$ and $\delta_{K}=0.1$. The Monte Carlo simulation method is carried out with 200 simulations. The confidence regions corresponding to a probability level $P_{c}=0.99$ are presented in Fig. 11 and Fig. 12. As it can be seen in these two figures, the sensitivity to uncertainties of local elastic modes is higher than for the global elastic modes. For observation Pobs 1 which is located in the stiff part of the structure and then, which is mainly driven by the global elastic modes, the main resonances are robust with respect to uncertainties. For observation Pobs 2 which is located in the flexible part of the structure and then, which is mainly driven by the local elastic modes, the responses are not 
robust with respect to uncertainties.

\section{Conclusions}

A general method has been developed and validated to construct a stochastic reduced-order model in low-frequency dynamics in presence of numerous local elastic modes. The projection basis is made up of two families of vector bases: the global elastic modes and the local elastic modes which are separately computed. The effects of uncertainties on the local modes are taken into account with the nonparametric probabilistic approach. The double projection method proposed is not really intrusive and can easily be implemented in commercial software.

\section{A Appendix: Proof concerning the construction of the basis of the admissible space}

In order to prove that the family $\left\{\varphi_{1}^{g}, \ldots, \varphi_{3 n_{J}}^{g}, \varphi_{1}^{\ell}, \varphi_{2}^{\ell}, \ldots\right\}$ is a basis of space $\mathbb{V}$, we have to prove (1) that for any finite integer $n_{\ell}$, the finite family of algebraically independent functions $\left\{\varphi_{1}^{g}, \ldots, \varphi_{3 n_{J}}^{g}\right\}$ is independent of the finite family of algebraically independent functions $\left\{\varphi_{1}^{\ell}, \ldots, \varphi_{n_{\ell}}^{\ell}\right\}$ and (2) that the family $\left\{\varphi_{1}^{g}, \ldots, \varphi_{3 n_{J}}^{g}, \varphi_{1}^{\ell}, \varphi_{2}^{\ell}, \ldots\right\}$ is total.

(1) In order to prove the independence of the two families, we have to prove that if $\sum_{\alpha=1}^{3 n_{J}} q_{\alpha}^{g} k\left(\varphi_{\alpha}^{g}, v\right)+\sum_{\alpha^{\prime}=1}^{n_{\ell}} q_{\alpha^{\prime}}^{\ell} k\left(\varphi_{\alpha^{\prime}}^{\ell}, v\right)=$ 0 for all $v$, then $q_{1}^{g}=\ldots=q_{3 n_{J}}^{g}=q_{1}^{\ell}=\ldots=q_{n_{\ell}}^{\ell}=0$. Using Eqs. (14) and (17) yields $\sum_{\alpha=1}^{3 n_{J}} q_{\alpha}^{g} \lambda_{\alpha}^{g} m^{r}\left(\varphi_{\alpha}^{g}, v\right)+\sum_{\alpha^{\prime}=1}^{n_{\ell}} q_{\alpha^{\prime}}^{\ell} \lambda_{\alpha^{\prime}}^{\ell} m^{c}\left(\varphi_{\alpha^{\prime}}^{\ell}, v\right)=$ 0 which can also be rewritten as $m\left(\sum_{\alpha=1}^{3 n_{J}} q_{\alpha}^{g} \lambda_{\alpha}^{g} h_{\varepsilon}^{r}\left(\varphi_{\alpha}^{g}\right)+\sum_{\alpha^{\prime}=1}^{n_{\ell}} q_{\alpha^{\prime}}^{\ell} \lambda_{\alpha^{\prime}}^{\ell} h_{\varepsilon}^{c}\left(\varphi_{\alpha^{\prime}}^{\ell}\right), v\right)=0$ for all $v$. Since $m$ is a positive-definite bilinear form, it can be deduced that $\sum_{\alpha=1}^{3 n_{J}} q_{\alpha}^{g} \lambda_{\alpha}^{g} h_{\varepsilon}^{r}\left(\varphi_{\alpha}^{g}\right)+\sum_{\alpha^{\prime}=1}^{n_{\ell}} q_{\alpha^{\prime}}^{\ell} \lambda_{\alpha^{\prime}}^{\ell} h_{\varepsilon}^{c}\left(\varphi_{\alpha^{\prime}}^{\ell}\right)=0$ which, due to $\mathbb{H}_{1}=\mathbb{H}_{r} \oplus \mathbb{H}_{c}$, yields $q_{1}^{g}=\ldots=q_{3 n_{J}}^{g}=q_{1}^{\ell}=\ldots=q_{n_{\ell}}^{\ell}=0$.

(2) In order to prove that the family is total, we have to prove that if $k\left(\varphi_{\alpha}^{g}, v\right)=0$ for all $\alpha=1, \ldots, 3 n_{J}$ and $k\left(\varphi_{\alpha^{\prime}}^{\ell}, v\right)=0$ for all $\alpha^{\prime}=1,2, \ldots$, then $v=0$. Taking into account Eqs. (14) and (17), its is equivalent to prove that if $\lambda_{\alpha}^{g} m^{r}\left(\varphi_{\alpha}^{g}, v\right)=0$ for all $\alpha=1, \ldots, 3 n_{J}$ and $\lambda_{\alpha^{\prime}}^{\ell} m^{c}\left(\varphi_{\alpha^{\prime}}^{\ell}, v\right)=0$ for all $\alpha^{\prime}=1,2, \ldots$, then we have $v=0$. Since $\lambda_{\alpha}^{g}$ and $\lambda_{\alpha^{\prime}}^{\ell}$ are strictly positive real numbers, we have to prove that $m^{r}\left(\varphi_{\alpha}^{g}, v\right)=0$ for all $\alpha=1, \ldots, 3 n_{J}$ and $m^{c}\left(\varphi_{\alpha^{\prime}}^{\ell}, v\right)=0$ for all $\alpha^{\prime}=1,2, \ldots$ implies $v=0$. Taking into account Eqs. (16) and (19), the first set of equations is verified for $v$ in $\mathbb{H}_{c}$ and the second set of equations is verified for $v$ in $\mathbb{H}_{r}$. Since $\mathbb{H}=\mathbb{H}_{r} \oplus \mathbb{H}_{c}$, there is only the zero solution $v=0$.

\section{References}

[1] Argyris, J., and Mlejnek, H. P., 1991, Dynamics of Structures, North-Holland, Amsterdam.

[2] Clough, R. W., and Penzien, J., 1975, Dynamics of Structures, McGraw-Hill, New York.

[3] Inman, D.J., 1989, "Vibration: With Control, Measurement, and Stability", Prentice-Hall, Englewood Cliffis, New Jersey.

[4] Meirovitch, L., 1990, Dynamics and Control of Structures, Wiley, New York.

[5] Morand, H. J. P., and Ohayon, R., 1995, Fluid Structure Interaction, Wiley, New York.

[6] Ohayon, R., and Soize, C., 1998, Structural Acoustics and Vibration, Academic Press, San Diego. 
[7] Hurty, W. C., 1965, "Dynamic Analysis of Structural Systems Using Component Modes”, AIAA Journal, 3(4), pp. 678685 .

[8] Craig, R., and Bampton, M., 1968, ”Coupling of Substructures for Dynamic Analyses”, AIAA J., 6(7), pp. 13131319.

[9] Mac Neal, R., 1971, ”A Hybrid Method of Component Mode Synthesis”, Comput. Struct., 1(4), pp. 581601.

[10] Rubin, S., 1975, ”Improved Component-Mode Representation for Structural Dynamic Analysis”, AIAA J., 13(8), pp. 9951006.

[11] Leung, A. Y. T., 1993, Dynamic Stiffness and Substructures, Springer-Verlag, New York.

[12] Farhat, C., and Geradin, M., 1994, ”On a Component Mode Synthesis Method and Its Application to Incompatible Substructures", Comput. Struct., 51(5), pp. 459473.

[13] Ohayon, R., Sampaio, R., and Soize, C., 1997, ”Dynamic Substructuring of Damped Structures Using Singular Value Decomposition”, Journal of Applied Mechanics - Transactions of the ASME, 64(2), pp. 292-298.

[14] Gawronski, W., 1998, Dynamics and control of structures, A modal approach, Springer-Verlag, New York.

[15] Skelton, R.E., 1988, Dynamic Systems Control, John Wiley and Sons, New York.

[16] Soize, C., 1986, ’Probabilistic Structural Modeling in Linear Dynamic Analysis of Complex Mechanical Systems, I Theoretical Elements”, La Recherche Aérospatiale (English edition), 5, pp. 23-48.

[17] Soize, C., 1993, ”A Model and Numerical Method in the Medium Frequency Range for Vibroacoustic Predictions Using Theory of Structural fuzzy”, J. Acoust. Soc. Am., 94(2), pp. 849-866.

[18] Sparrow, V.W., Russel, D.A., and Rochat, J.L., 1994, "Implementation of Discrete Fuzzy Structure Models in Mathematica”, Int. J. Numer. Methods Eng., 37, pp. 3005-3014.

[19] Russel, D.A., and Sparrow, V.W., 1995, ”Backscattering From a Baffled Finite Plate Strip With Fuzzy Attachments”, J. Acoust. Soc. Am., 98(3), pp. 1527-533.

[20] Pierce, A.D., Sparrow, V.W. and Russel, D.A., 1995, ’Fundamental Structural-Acoustic Idealizations for Structures with Fuzzy Internal”, Journal of Vibration and Acoustics, 117, pp. 339-348.

[21] Maidanik, G., 1995, ’Power Dissipation in a Sprung Mass Attached to a Master Structure”, J. Acoust. Soc. Am, 98(3), pp. 3527-3533.

[22] Strasberg, M., and Feit, D., 1996, ”Vibration Damping of Large Structures Induced by Attached Small Resonant Structures", J. Acoust. Soc. Am., 99(1), pp. 335-344.

[23] Weaver, R.L., 1996, "The Effect of an Undamped Finite Degree of Freedom Fuzzy Substructure: Numerical Solutions and Theoretical Discussion”, J. Acoust. Soc. Am., 100(5), pp. 3159-3164.

[24] Langley, R.S., and Bremmer, P., 1999, "A Hybrid Method for the Vibration Analysis of Complex Structural-Acoustic Systems", J. Acoust. Soc. Am. 105(3), 1657-1672.

[25] Friis, L., and Ohlrich, M., 2008, "Vibration Modeling of Structural Fuzzy with Continuous Boundary", J. Acoust. Soc. Am., 123(2), pp. 718-728.

[26] Fernandez, C., Soize, C., and Gagliardini, L., 2009, ”Fuzzy Structure Theory Modeling of Sound-Insulation Layers in Complex Vibroacoustic Uncertain Sytems - Theory and Experimental Validation”, J. Acoust. Soc. Am., 125(1), pp. 
$138-153$

[27] Bucher, I. and Braun, S.G., 1997, "Left Eigenvectors: Extraction From Measurements and Physical Interpretation”, Journal of Applied Mechanics - Transactions of the ASME, 64(1), pp. 97-105.

[28] Hansen, P.C., 1987, ’The Truncated SVD as a Method for Regularization”, B.LT., 28, pp. 543-553.

[29] Bathe, K.J., and Wilson, E.L., 1976, Numerical Methods in the Finite Element Method, Prentice-Hall, Englewood Cliffs, New Jersey

[30] Zienkiewicz, O.C., and Taylor, R.L., 2000, The Finite Element Method, Fifth edition, Butterworth-Heinemann, Oxford

[31] Chan, H.C., Cai, C.W., and Cheung, Y.K., 1993, "Convergence Studies of Dynamic Analysis by Using the Finite Element Method with Lumped Mass Matrix”, Journal of Sound and Vibration, 165(2), pp. 193-207.

[32] Jensen, M.S., 1996, "High Convergence Order Finite Elements With Lumped Mass Matrix”, International Journal for Numerical Methods in Engineering, 39(11), pp. 1879-1888.

[33] Belytschko, T., and Mindle, W.L., 1980, "Flexural Wave-propagation Behavior of Lumped Mass Approximations", Computer and Structures, 12(6), pp. 805-812.

[34] Guyan, R. J., 1965, ” Reduction of Stiffness and Mass Matrices”, AIAA Journal, 3, pp. 380-388.

[35] Schueller, G.I., 2005, "Computational Methods in Stochastic Mechanics and Reliability Analysis”, Comput. Methods Appl. Mech. Eng., 194(1216), pp. 12511795.

[36] Soize, C., 2000, "A Nonparametric Model of Random Uncertainties on Reduced Matrix Model in Structural Dynamics", Probabilistic Engineering Mechanics, 15(3), pp. 277-294.

[37] Soize, C., 2005, "Random Matrix Theory for Modeling Random Uncertainties in Computational Mechanics", Comput. Methods Appl. Mech. Eng., 194(1216), pp. 13331366.

[38] Chebli, H., and Soize, C., 2004, ’Experimental Validation of a Nonparametric Probabilistic Model of Non Homogeneous Uncertainties for Dynamical Systems", J. Acoust. Soc. Am., 115(2), pp. 697-705.

[39] Chen, C., Duhamel, D., and Soize, C., 2006, "Probabilistic Approach for Model and Data Uncertainties and Its Experimental Identification in Structural Dynamics: Case of Composite Sandwich Panels, Journal of Sound and Vibration, 294(12), pp. 6481.

[40] Duchereau, J., and Soize, C., 2006, ”Transient Dynamics in Structures with Nonhomogeneous Uncertainties Induced by Complex Joints”, Mechanical Systems and Signal Processing, 20(4), pp. 854-867.

[41] Durand, J.-F., Soize, C., and Gagliardini, L., 2008, ”Structural-Acoustic Modeling of Automotive Vehicles in Presence of Uncertainties and Experimental Identification and Validation”, J. Acoust. Soc. Am., 124(3), pp. 1513-1525.

[42] Shannon, C.E., 1948, ”A Mathematical Theory of Communication”, Bell System Technology Journal, 27, pp. 379-423 and pp. 623-659.

[43] Jaynes, E.T., 1957, ”Information Theory and Statistical Mechanics”, Physical Review, 106 (4), pp. $620-630$ and 108 (2), pp. 171-190. 


\section{LIST OF FIGURES CAPTIONS}

Fig 1: Geometry of the dynamical system.

Fig 2: First elastic mode.

Fig 3: Second elastic mode.

Fig 4: Distribution of the number of eigenfrequencies for the global elastic modes (black histogram) and for the local elastic modes (grey histogram) as a function of the frequency in $\mathrm{Hz}$.

Fig 5: Elastic mode 1.

Fig 6: Elastic mode 2.

Fig 7: Elastic mode 3.

Fig 8: Elastic mode 4.

Fig 9: Modulus in log scale of the frequency response for Pobs ${ }_{1}$. Comparisons between different projection bases: initial elastic modes (solid thick line), global elastic modes only (mixed line), local elastic modes only (dashed line), global and local elastic modes (solid thin line superimposed to the solid thick line).

Fig 10: Modulus in log scale of the frequency response for Pobs 2 . Comparisons between different projection bases: initial elastic modes (solid thick line), global elastic modes only (mixed line), local elastic modes only (dashed line), global and local elastic modes (solid thin line superimposed to the solid thick line).

Fig 11: Random frequency response functions for Pobs ${ }_{1}$. Confidence region (lower and upper lines), mean response (middle line), deterministic response calculated with the initial elastic modes (solid thick line).

Fig 12: Random frequency response functions for Pobs 2 . Confidence region (lower and upper lines), mean response (middle line), deterministic response calculated with the initial elastic modes (solid thick line). 
FIGURES

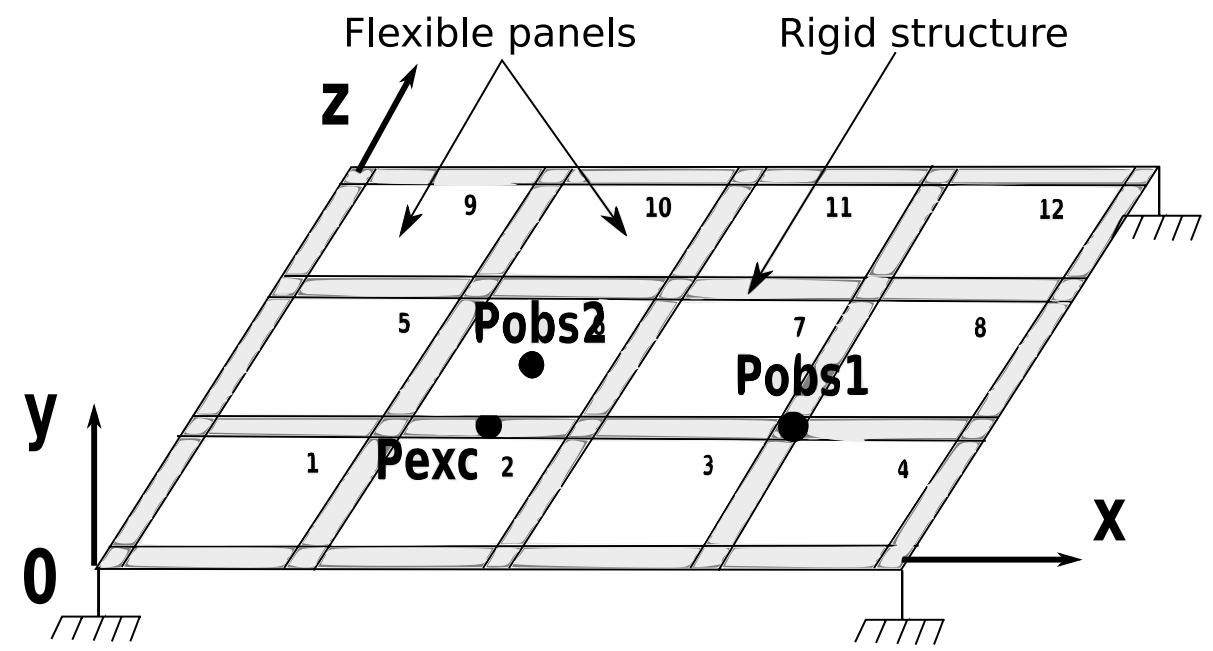

Fig. 1. fig1.eps. 


\section{Mode 1 - $1.6722 \mathrm{~Hz}$}

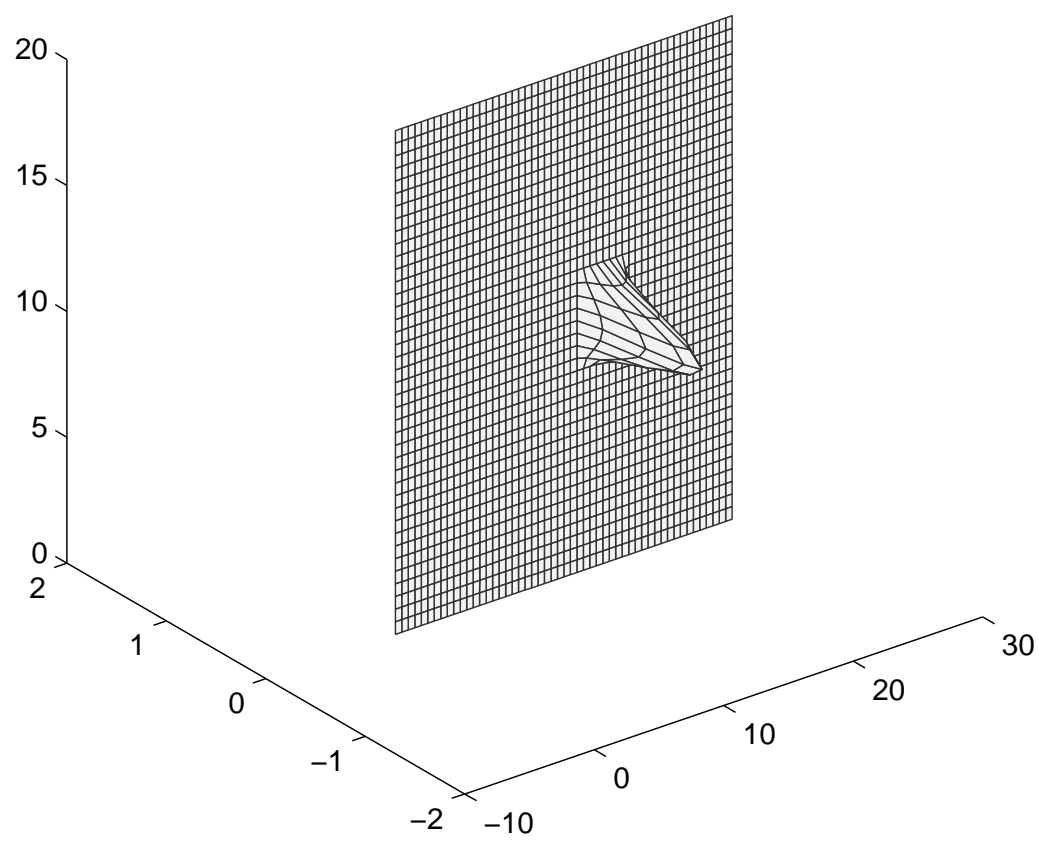

Fig. 2. fig2.eps. 


\section{Mode $21.8204 \mathrm{~Hz}$}

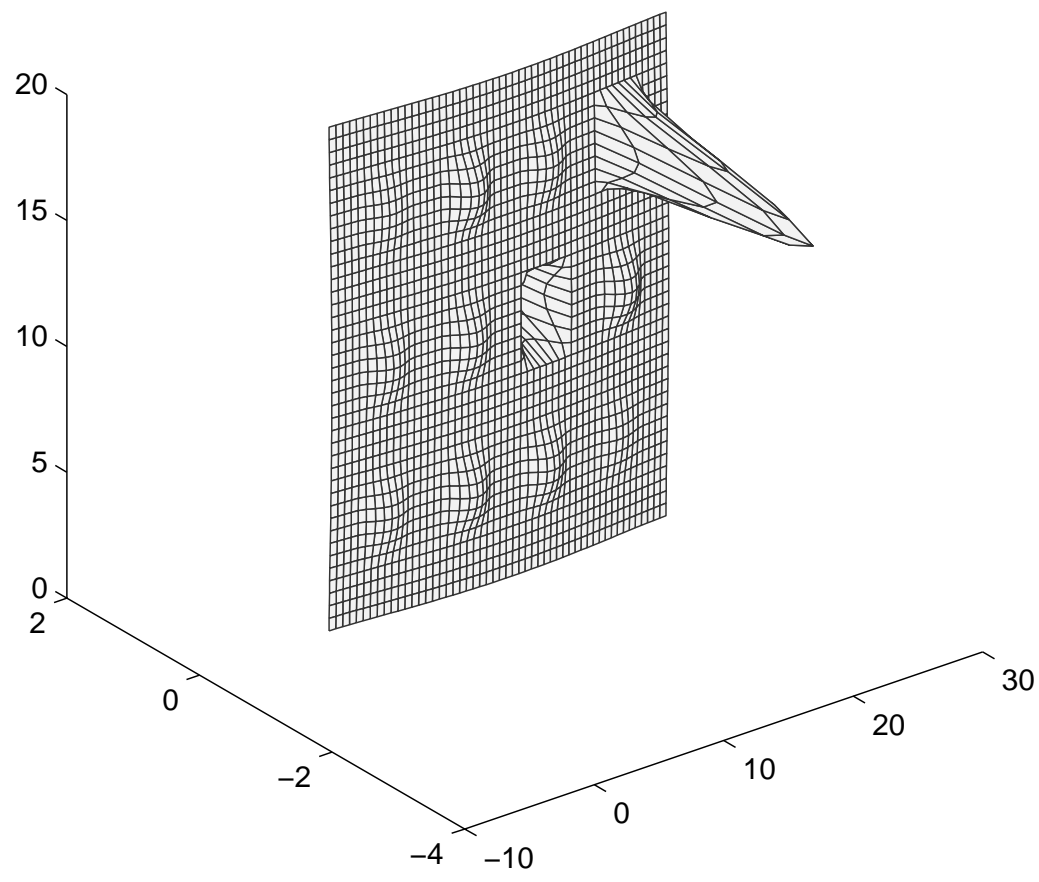

Fig. 3. fig3.eps. 


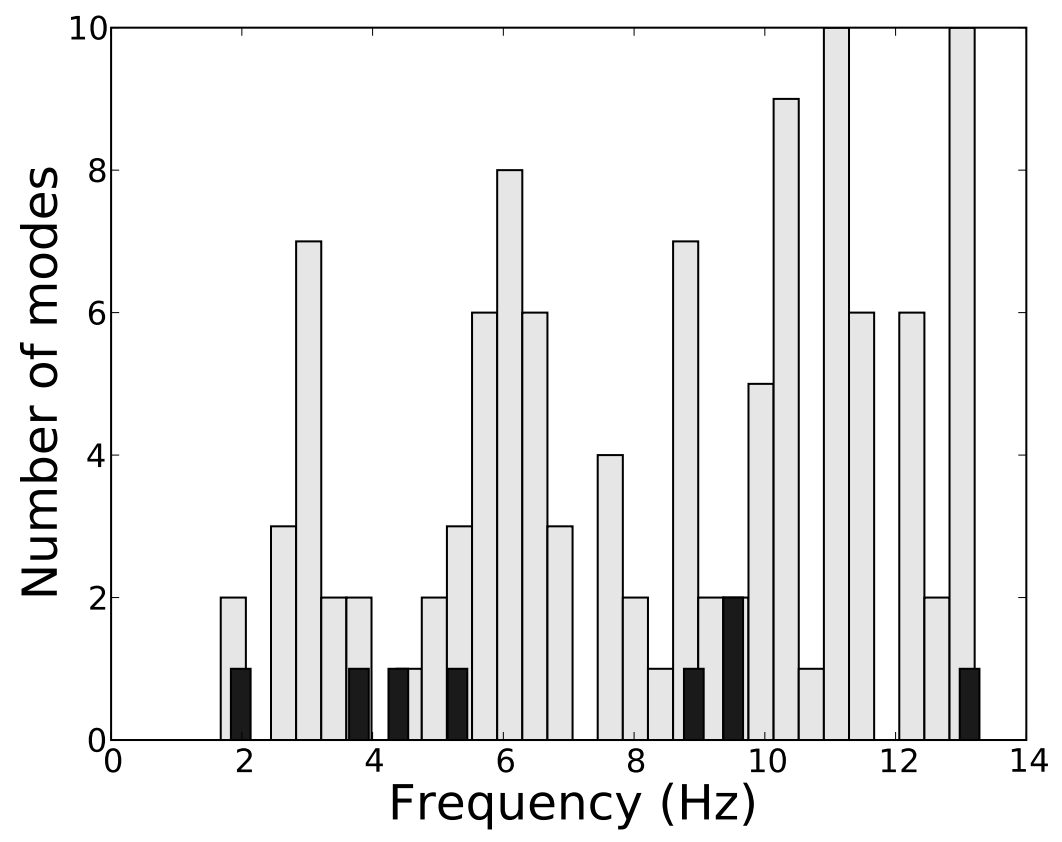

Fig. 4. fig4.eps. 


\section{Mode 1 - $1.8325 \mathrm{~Hz}$}

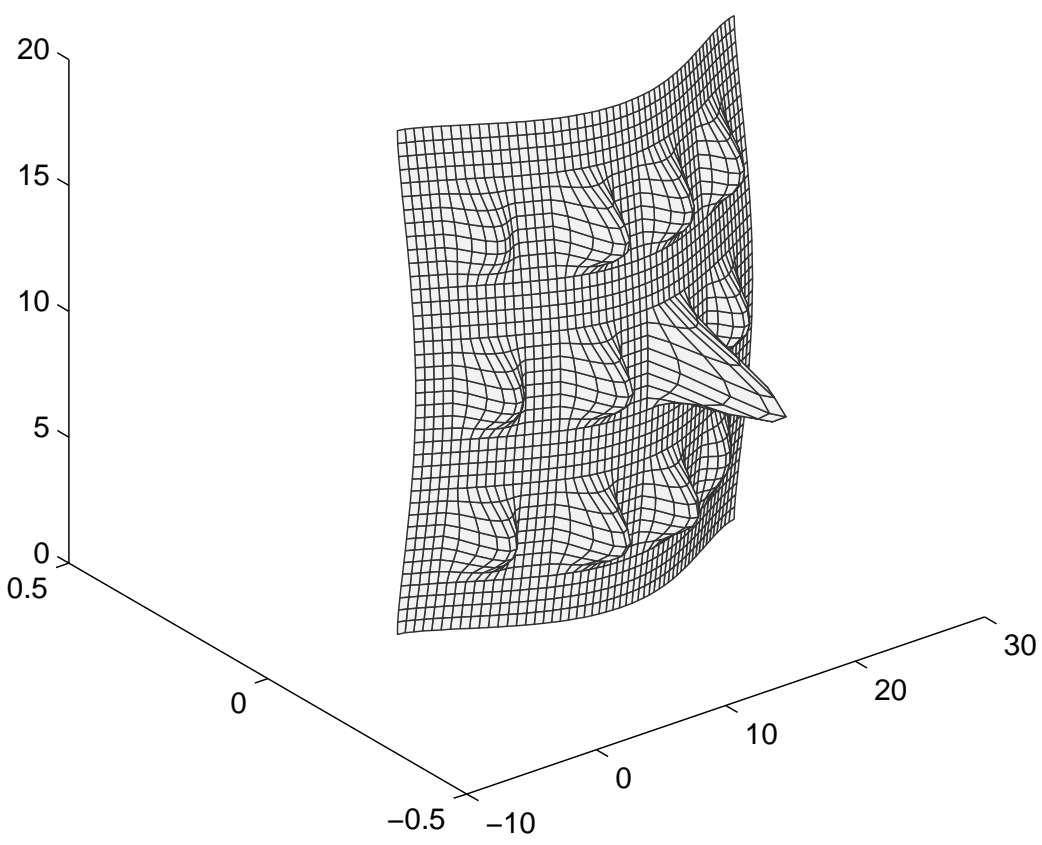

Fig. 5. fig5.eps. 


\section{Mode 2 - $3.8122 \mathrm{~Hz}$}

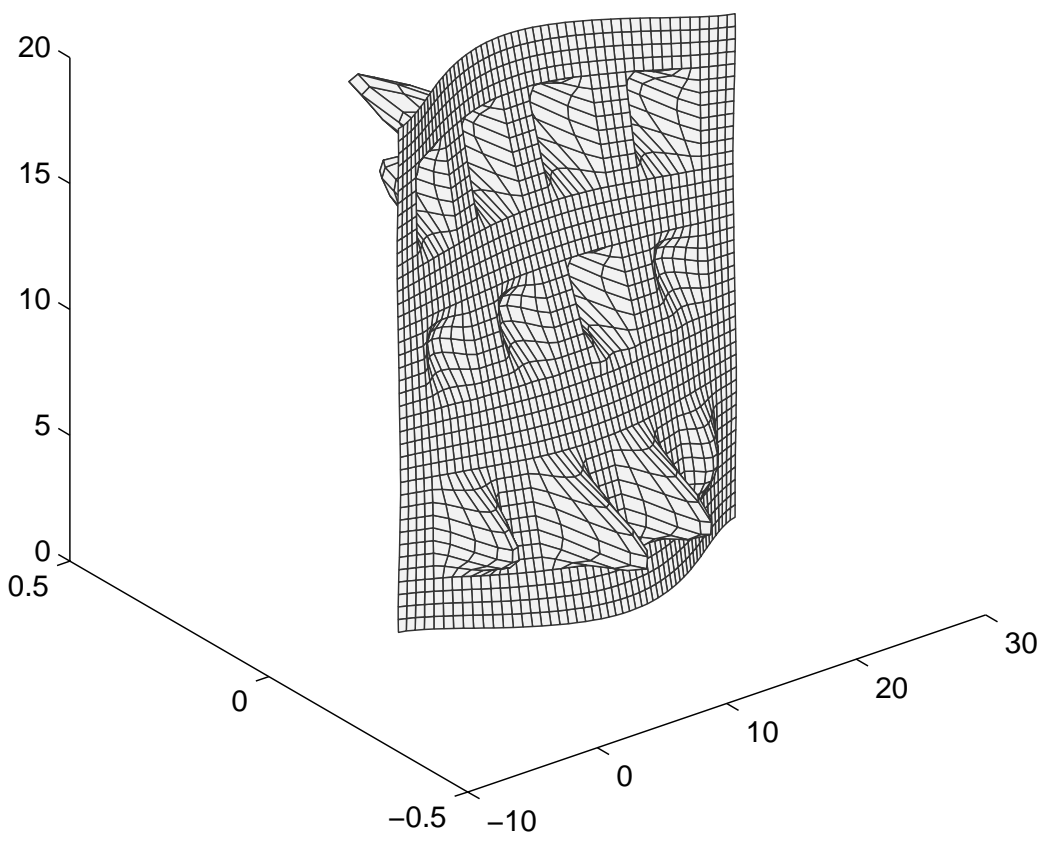

Fig. 6. fig6.eps. 
Mode $3-4.4267 \mathrm{~Hz}$

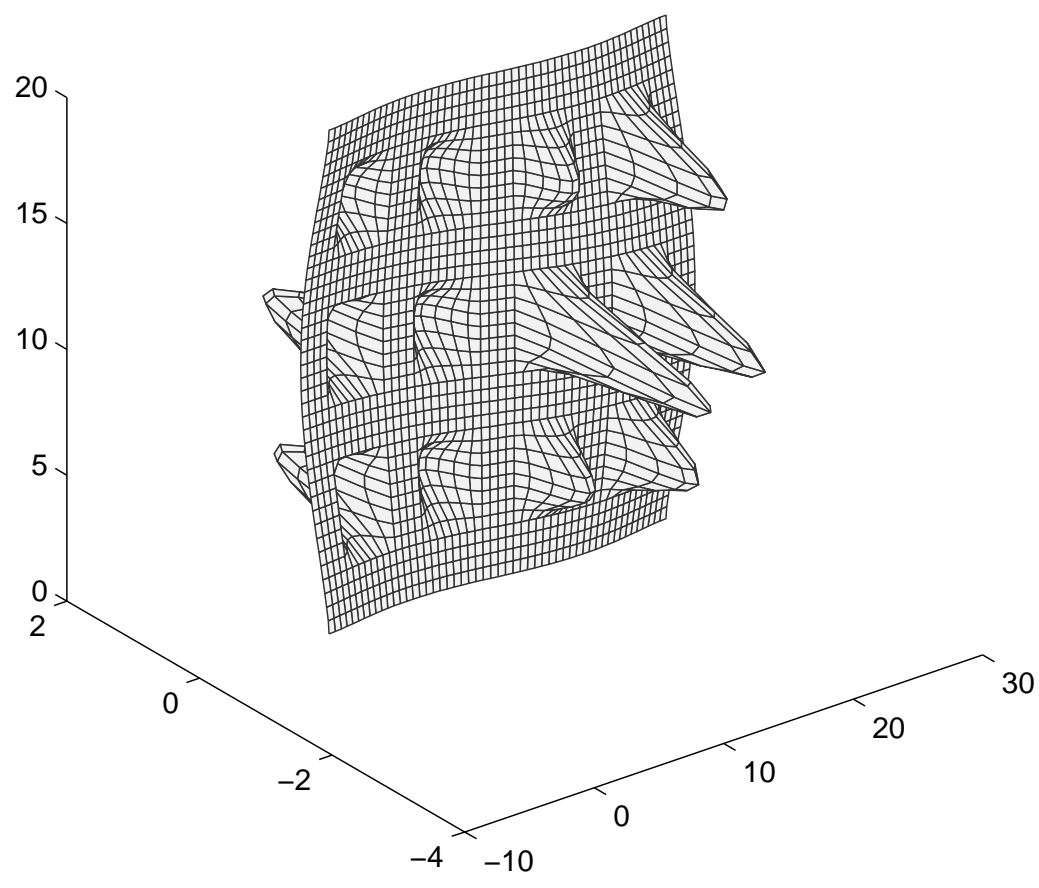

Fig. 7. fig7.eps. 


\section{Mode 4 - $5.2123 \mathrm{~Hz}$}

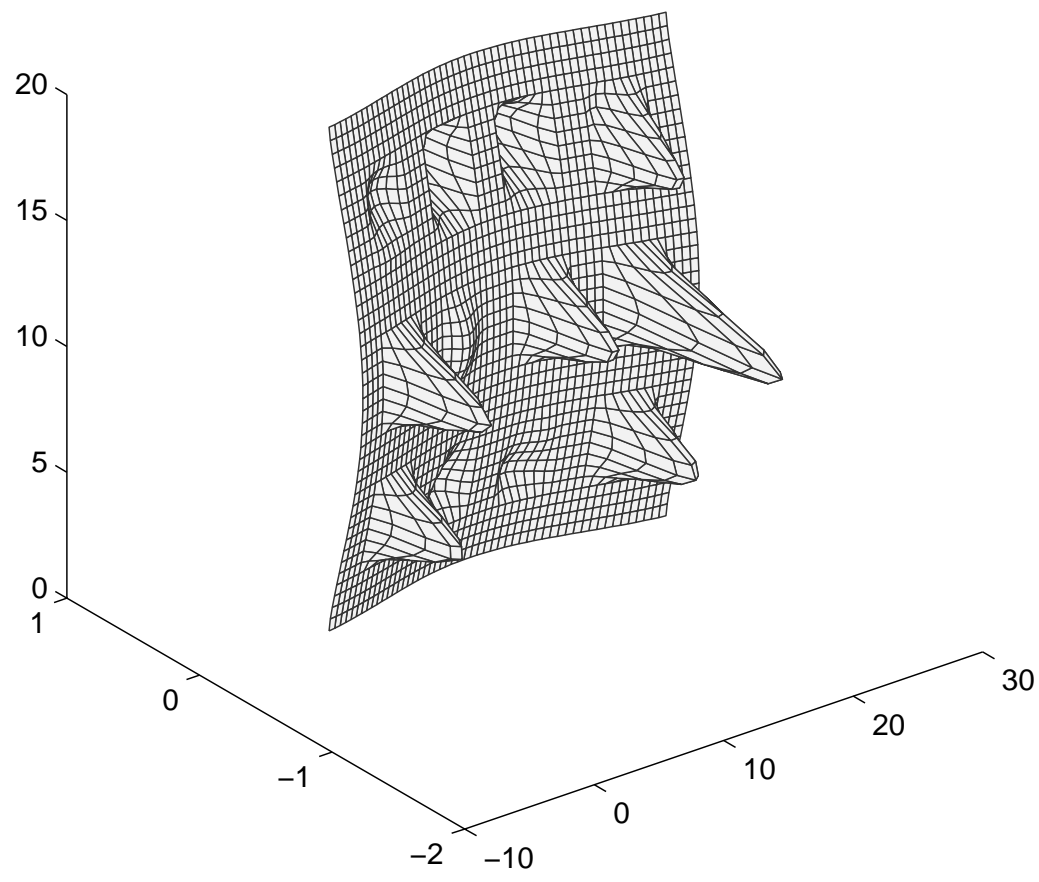

Fig. 8. fig8.eps. 


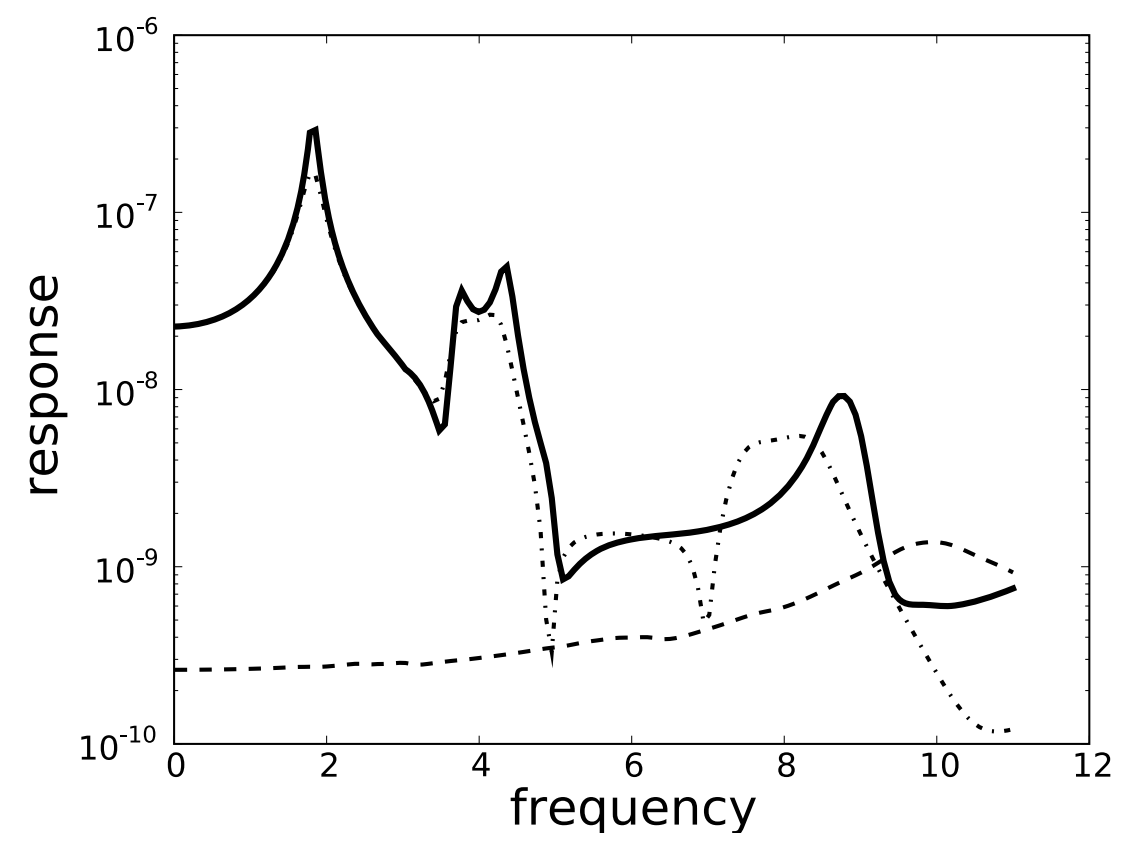

Fig. 9. fig9.eps. 


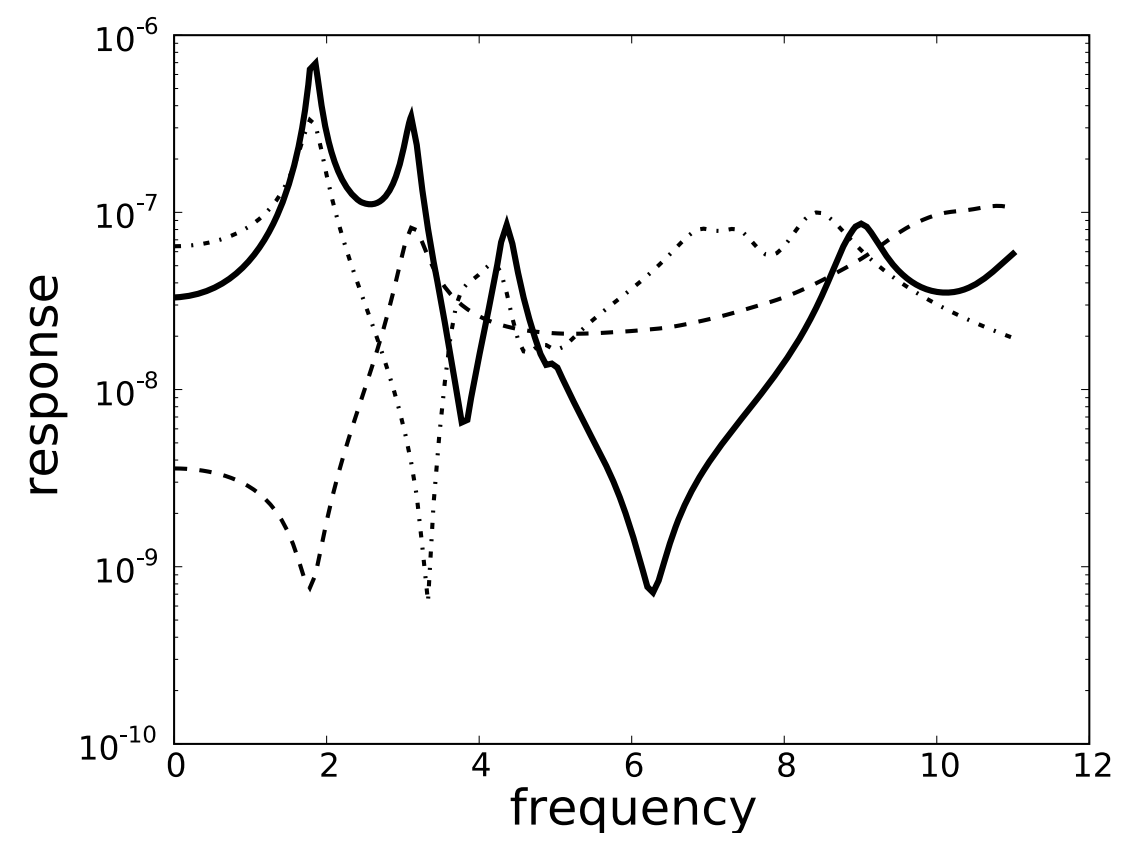

Fig. 10. fig10.eps. 


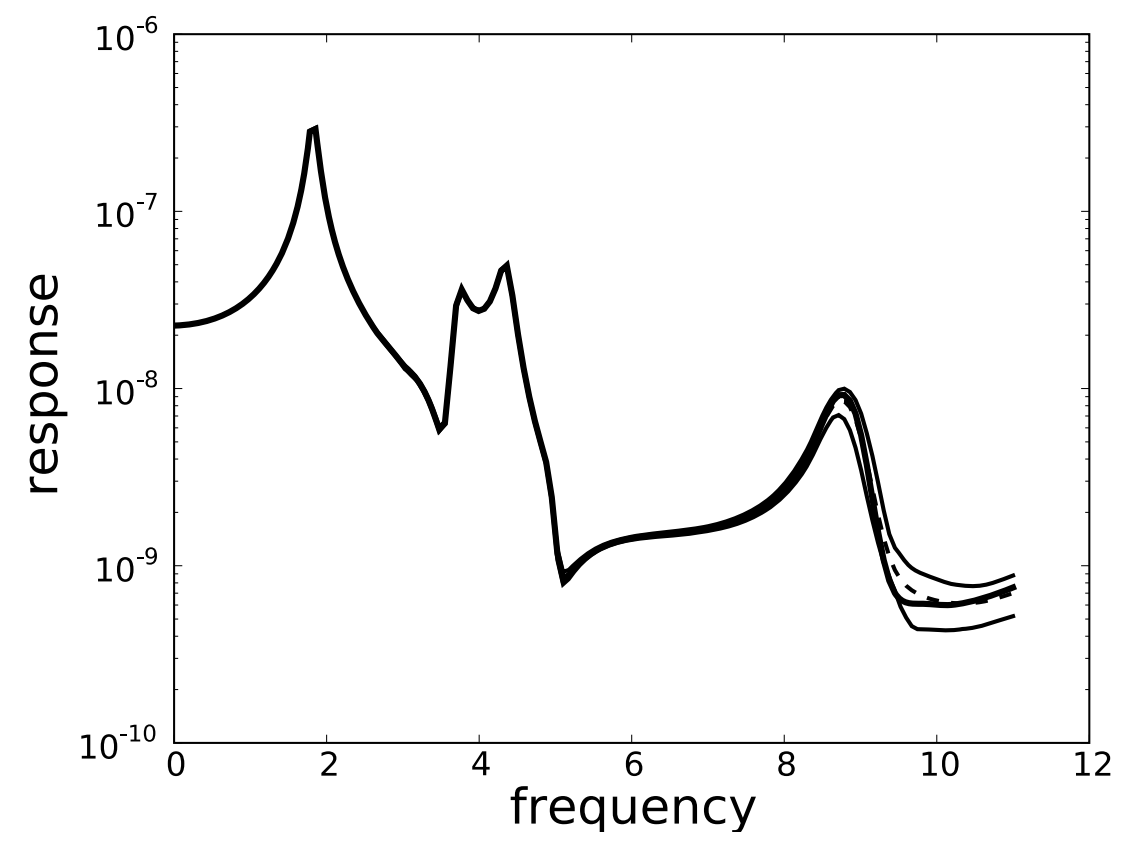

Fig. 11. fig11.eps. 


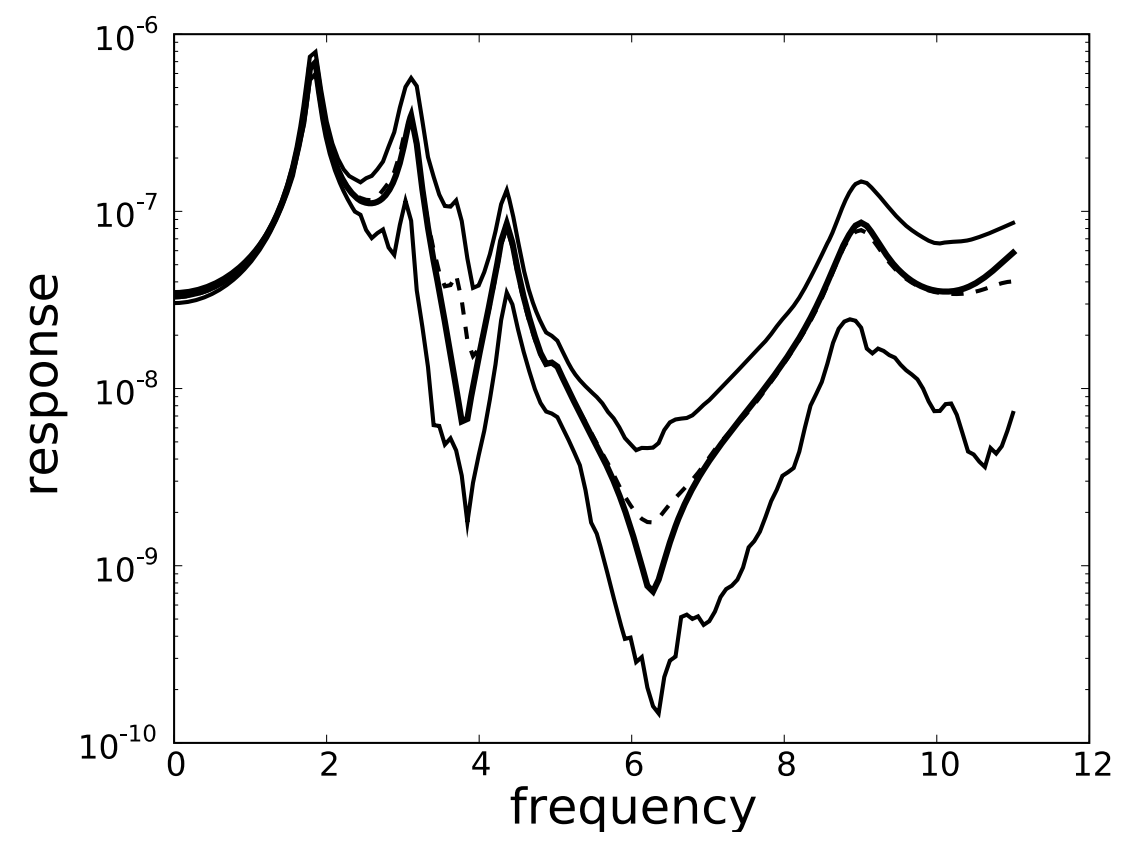

Fig. 12. fig12.eps. 